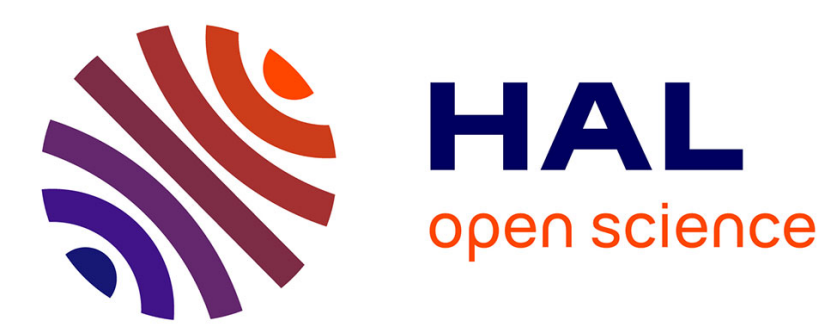

\title{
On the Navier-Stokes system with the Coulomb friction law boundary condition
}

\author{
Loredana Bălilescu, Jorge San Martín, Takéo Takahashi
}

\section{To cite this version:}

Loredana Bălilescu, Jorge San Martín, Takéo Takahashi. On the Navier-Stokes system with the Coulomb friction law boundary condition. Zeitschrift für Angewandte Mathematik und Physik, 2017, 68 (3), 10.1007/s00033-016-0744-x . hal-01393709

\section{HAL Id: hal-01393709 \\ https://hal.science/hal-01393709}

Submitted on 16 Nov 2016

HAL is a multi-disciplinary open access archive for the deposit and dissemination of scientific research documents, whether they are published or not. The documents may come from teaching and research institutions in France or abroad, or from public or private research centers.
L'archive ouverte pluridisciplinaire HAL, est destinée au dépôt et à la diffusion de documents scientifiques de niveau recherche, publiés ou non, émanant des établissements d'enseignement et de recherche français ou étrangers, des laboratoires publics ou privés. 


\title{
ON THE NAVIER-STOKES SYSTEM WITH THE COULOMB FRICTION LAW BOUNDARY CONDITION
}

\author{
LOREDANA BĂLILESCU ${ }^{\S} *$, JORGE SAN MARTÍN${ }^{\ddagger}$, AND TAKÉO TAKAHASHI ${ }^{\dagger}$
}

\begin{abstract}
We propose a new model for the motion of a viscous incompressible fluid. More precisely, we consider the Navier-Stokes system with a boundary condition governed by the Coulomb friction law. With this boundary condition, the fluid can slip on the boundary if the tangential component of the stress tensor is too large. We prove the existence and uniqueness of weak solution in the two-dimensional problem and the existence of at least one solution in the three-dimensional case, together with regularity properties and an energy estimate. We also propose a fully discrete scheme of our problem using the characteristic method and we present numerical simulations in two physical examples.
\end{abstract}

\section{InTRODUCTION}

In this paper, we analyze the existence and uniqueness of solutions for the Navier-Stokes system when the boundary condition is governed by the Coulomb friction law. We recall that the classical results of existence and uniqueness of solutions for the Navier-Stokes system with Dirichlet or Neumann boundary conditions can be found in the literature in many publications, as for instance [9, 17, 10]. In previous works [2, 15] and references therein, these results have been extended to the case of solid-fluid interactions. In [15], the authors have obtained a non-intuitive result, which asserts that two rigid solids can't collide if they are surrounded by a viscous incompressible fluid. In that result, a key ingredient is the nonslip boundary condition. In order to get a more realistic model for this situations, the authors in [4] studied the model with the so-called Navier boundary condition and after that, in [5] they proved that with this boundary condition, the solid can collide with the boundary. In our paper, we propose to study the existence of weak solutions for the Navier-Stokes system when we impose the Coulomb friction law as a boundary condition. This boundary condition seems to be more natural since, for small tangential stresses, it gives the non-slip boundary condition and after a certain threshold the fluid can slip at the boundary.

In order to write this new boundary condition we use the technique of subdifferential. In Section 2 we describe the model using this technique and we state the main result of the paper. Section 3 is devoted to prove the main result. Finally, in Section 4 we propose a numerical scheme and we present simulations in order to show the influence of this boundary condition on two physical situations: an abrupt contraction and the vortices after a cylindrical obstacle.

$\S$ Department of Mathematics, Federal University of Santa Catarina, Brasil and Department of Mathematics and Computer Science, University of Piteşti, 110040 Piteşti, Str. Târgu din Vale nr.1, Argeş, Romania; smaranda@dim.uchile.cl

$\ddagger$ Departamento de Ingeniería Matemática, Facultad de Ciencias Físicas y Matemáticas, Universidad de Chile and Centro de Modelamiento Matemático, UMR 2071 CNRS-UChile and Centro de Biotecnología \& Bioingeniería, Casilla 170/3 - Correo 3, Santiago, Chile; jorge@dim.uchile.cl

$\dagger$ Inria, Villers-lès-Nancy F-54600, France and University of Lorraine, Vandœuvre-lès-Nancy 54506, France; takeo.takahashi@inria.fr

* Corresponding author. 


\section{Model Description And Main Result}

We consider a viscous incompressible fluid that occupies an open bounded domain $\Omega \subset \mathbb{R}^{d}$, with $d=2$ or $d=3$, where the boundary of $\Omega$ is locally Lipschitz. The Eulerian velocity field $\mathbf{u}$ and the pressure field $p$ of the fluid satisfy the following Navier-Stokes system:

$$
\begin{aligned}
\frac{\partial \mathbf{u}}{\partial t}+(\mathbf{u} \cdot \nabla) \mathbf{u}-\operatorname{div} \sigma(\mathbf{u}, p)=0 & \text { in } \Omega, \\
\operatorname{div} \mathbf{u}=0 & \text { in } \Omega,
\end{aligned}
$$

where $\sigma$ denotes the stress tensor field. Using the classical notation $\mathbf{D}(\mathbf{u})=\frac{1}{2}\left((\nabla \mathbf{u})+(\nabla \mathbf{u})^{T}\right)$, $\sigma$ is defined by

$$
\sigma(\mathbf{u}, p)=2 \mu D(\mathbf{u})-p \mathrm{Id},
$$

with Id the identity matrix in $M_{d}(\mathbb{R})$ and $\mu>0$ the dynamic viscosity of the fluid, which is supposed to be a constant.

In order to describe the boundary conditions considered in this paper, we introduce some additional notation. If we denote by $\mathbf{n}:=\mathbf{n}(\mathbf{x})$ the exterior unit normal of $\partial \Omega$, we can decompose any vector $\mathbf{a} \in \mathbb{R}^{d}$ as follows:

$$
\mathbf{a}=(\mathbf{a} \cdot \mathbf{n}) \mathbf{n}+(\mathbf{a}-(\mathbf{a} \cdot \mathbf{n}) \mathbf{n}) .
$$

Each component of this decomposition is denoted by $\mathbf{a}_{n}$, respectively $\mathbf{a}_{\tau}$. That is,

$$
\mathbf{a}_{n}:=(\mathbf{a} \cdot \mathbf{n}) \mathbf{n}, \quad \mathbf{a}_{\tau}:=\mathbf{a}-(\mathbf{a} \cdot \mathbf{n}) \mathbf{n} \quad \text { and } \quad \mathbf{a}=\mathbf{a}_{n}+\mathbf{a}_{\tau} .
$$

Using this notation, we are now able to describe the Coulomb friction law. The velocity field $\mathbf{u}$ and the normal stress tensor $\sigma(\mathbf{u}, p) \mathbf{n}$ on the boundary $\partial \Omega$ are decomposed in accordance with (5). We first impose that the normal component of the fluid velocity $\mathbf{u}_{n}$ is equal to 0 . Secondly, for the tangential components, we assume that there exists a physical constant $g>0$ such that if $\left|(\sigma(\mathbf{u}, p) \mathbf{n})_{\tau}\right|<g$ then $\mathbf{u}_{\tau}=0$ and if $\left|(\sigma(\mathbf{u}, p) \mathbf{n})_{\tau}\right|=g$ then $\mathbf{u}_{\tau}$ has the same direction and sense with $-(\sigma(\mathbf{u}, p) \mathbf{n})_{\tau}$. This boundary condition is known in the literature as the Coulomb friction law (or dry friction law). We now use the classical convex theory applied to mechanics and physics (see for instance [3, 7]), in order to show that this boundary condition can be written as follows

$$
\begin{aligned}
\mathbf{u}_{n}=0 & \text { on } \partial \Omega, \\
-\mathbf{u}_{\tau} \in \partial I_{B(0, g)}\left((\sigma(\mathbf{u}, p) \mathbf{n})_{\tau}\right) & \text { on } \partial \Omega,
\end{aligned}
$$

where $I_{B(0, g)}$ denotes the indicator function of closed convex ball $B(0, g)$ and is defined by

$$
\begin{aligned}
I_{B(0, g)}: \mathbb{R}^{d} & \rightarrow \mathbb{R} \cup\{+\infty\} \\
\mathbf{x} & \mapsto I_{B(0, g)}(\mathbf{x})= \begin{cases}0 & \text { if }|\mathbf{x}| \leq g \\
+\infty & \text { if }|\mathbf{x}|>g\end{cases}
\end{aligned}
$$

Moreover, $\partial I_{B(0, g)}\left(\mathbf{x}_{0}\right)$ denotes the set of all subgradients at $\mathbf{x}_{0}$ of function $I_{B(0, g)}$, which is defined by

$$
\mathbf{y} \in \partial I_{B(0, g)}\left(\mathbf{x}_{0}\right) \Longleftrightarrow I_{B(0, g)}\left(\mathbf{x}_{0}\right)+\mathbf{y} \cdot\left(\mathbf{x}-\mathbf{x}_{0}\right) \leq I_{B(0, g)}(\mathbf{x}) \quad \forall \mathbf{x} \in \mathbb{R}^{d}
$$

In order to rewrite the condition (17), we first note that definition (9) could be written as follows

$$
\mathbf{y} \in \partial I_{B(0, g)}\left(\mathbf{x}_{0}\right) \Longleftrightarrow I_{B(0, g)}\left(\mathbf{x}_{0}\right)+\mathbf{y} \cdot\left(\mathbf{x}-\mathbf{x}_{0}\right) \leq 0 \quad \forall \mathbf{x} \in B(0, g) .
$$

Using relation (10) we deduce that if $\mathbf{x}_{0} \notin B(0, g)$, then $\partial I_{B(0, g)}\left(\mathbf{x}_{0}\right)$ is the empty set. For $\mathbf{x}_{0} \in \operatorname{Int} B(0, g)$, we have $\mathbf{y}=0$. Finally, for any $\mathbf{x}_{0}$ such that $\left|\mathbf{x}_{0}\right|=g$, we get that $\mathbf{y}$ belongs 
to the normal cone of $B(0, g)$ in $\mathbf{x}_{0}$, then there exists $\alpha \geq 0$ such that $\mathbf{y}=\alpha \mathbf{x}_{0}$. Due to the above remarks, we deduce that the boundary condition (17) is equivalent to the following relations

$$
\left|(\sigma(\mathbf{u}, p) \mathbf{n})_{\tau}\right| \leq g
$$

and

$$
\mathbf{u}_{\tau}= \begin{cases}0 & \text { if }\left|(\sigma(\mathbf{u}, p) \mathbf{n})_{\tau}\right|<g, \\ -\alpha(\sigma(\mathbf{u}, p) \mathbf{n})_{\tau} & \text { if }\left|(\sigma(\mathbf{u}, p) \mathbf{n})_{\tau}\right|=g, \text { where } \alpha \geq 0 .\end{cases}
$$

Thus, relations (11) -(12) are exactly the classical expressions of the Coulomb friction law described above.

Let us now use again results of convex analysis in order to transform the condition (7) into a global inequality. We begin by recalling that, since $I_{B(0, g)}$ is a lower semi-continuous function, then (7) is equivalent to

$$
-(\sigma(\mathbf{u}, p) \mathbf{n})_{\tau} \in \partial I_{B(0, g)}^{*}\left(\mathbf{u}_{\tau}\right),
$$

where $I_{B(0, g)}^{*}$ represents the conjugate function of $I_{B(0, g)}$ with respect to the inner product in $\mathbb{R}^{d}$, which is defined by

$$
I_{B(0, g)}^{*}(\mathbf{y})=\sup _{\mathbf{x} \in \mathbb{R}^{d}}\left\{\mathbf{y} \cdot \mathbf{x}-I_{B(0, g)}(\mathbf{x})\right\} \quad \forall \mathbf{y} \in \mathbb{R}^{d} .
$$

Simple computation yields to

$$
I_{B(0, g)}^{*}(\mathbf{y})=\sup _{\mathbf{x} \in B(0, g)} \mathbf{y} \cdot \mathbf{x}=\sup _{\mathbf{x} \in B(0,1)} g \mathbf{y} \cdot \mathbf{x}=g|\mathbf{y}| \quad \forall \mathbf{y} \in \mathbb{R}^{d} .
$$

Due to these relations, the boundary condition (17) can be written as follows

$$
(\sigma(\mathbf{u}, p) \mathbf{n})_{\tau} \cdot \mathbf{y} \geq g\left|\mathbf{u}_{\tau}\right|-g\left|\mathbf{u}_{\tau}+\mathbf{y}\right| \quad \forall \mathbf{y} \in \mathbb{R}^{d} .
$$

We can now rewrite the complete system that we are interested in considering also the corresponding initial data. Precisely,

$$
\begin{aligned}
\frac{\partial \mathbf{u}}{\partial t}+(\mathbf{u} \cdot \nabla) \mathbf{u}-\operatorname{div} \sigma(\mathbf{u}, p)=0 & \text { in } \Omega, \\
\operatorname{div} \mathbf{u}=0 & \text { in } \Omega, \\
\mathbf{u}_{n}=0 & \text { on } \partial \Omega, \\
(\sigma(\mathbf{u}, p) \mathbf{n})_{\tau} \cdot \mathbf{y} \geq g\left|\mathbf{u}_{\tau}\right|-g\left|\mathbf{u}_{\tau}+\mathbf{y}\right| & \text { on } \partial \Omega, \forall \mathbf{y} \in \mathbb{R}^{d}, \\
\mathbf{u}(\mathbf{x}, 0)=\mathbf{u}^{0}(\mathbf{x}) & \forall \mathbf{x} \in \Omega .
\end{aligned}
$$

Let us introduce the weak formulation of system (17)-(21). To this end, we consider the following Hilbert spaces:

$$
\begin{aligned}
H & =\left\{\mathbf{v} \in L^{2}(\Omega)^{d}: \operatorname{div} \mathbf{v}=0, \mathbf{v}_{n}=0 \text { on } \partial \Omega\right\}, \\
V & =\left\{\mathbf{v} \in H^{1}(\Omega)^{d}: \operatorname{div} \mathbf{v}=0, \mathbf{v}_{n}=0 \text { on } \partial \Omega\right\},
\end{aligned}
$$

where $L^{2}(\Omega)$ and $H^{1}(\Omega)$ are the classical Lebesgue and Sobolev spaces defined in [1, Chapters 4 and 9]. Let us denote by $V^{\prime}$ the dual space of $V$ with respect to $H$.

For any $\mathbf{v} \in V$, we multiply equation (17) by $\mathbf{v}$, we integrate by parts and we use the definition (3) to get

$$
\int_{\Omega}\left(\frac{\partial \mathbf{u}}{\partial t}+(\mathbf{u} \cdot \nabla) \mathbf{u}\right) \cdot \mathbf{v} d \mathbf{x}+2 \mu \int_{\Omega} D(\mathbf{u}): D(\mathbf{v}) d \mathbf{x}=\int_{\partial \Omega} \sigma(\mathbf{u}, p) \mathbf{n} \cdot \mathbf{v} d \Gamma
$$


Since $\mathbf{v}_{n}=0$, using decomposition (15), we have

$$
\int_{\Omega}\left(\frac{\partial \mathbf{u}}{\partial t}+(\mathbf{u} \cdot \nabla) \mathbf{u}\right) \cdot \mathbf{v} d \mathbf{x}+2 \mu \int_{\Omega} D(\mathbf{u}): D(\mathbf{v}) d \mathbf{x}=\int_{\partial \Omega}(\sigma(\mathbf{u}, p) \mathbf{n})_{\tau} \cdot \mathbf{v} d \Gamma .
$$

Using inequality (20) and the fact that $\mathbf{u}_{n}=0$, we get

$$
\int_{\Omega}\left(\frac{\partial \mathbf{u}}{\partial t}+(\mathbf{u} \cdot \nabla) \mathbf{u}\right) \cdot \mathbf{v} d \mathbf{x}+2 \mu \int_{\Omega} D(\mathbf{u}): D(\mathbf{v}) d \mathbf{x} \geq \int_{\partial \Omega}(g|\mathbf{u}|-g|\mathbf{u}+\mathbf{v}|) d \Gamma
$$

that is

$$
\int_{\Omega}\left(\frac{\partial \mathbf{u}}{\partial t}+(\mathbf{u} \cdot \nabla) \mathbf{u}\right) \cdot \mathbf{v} d \mathbf{x}+a(\mathbf{u}, \mathbf{v})+J(\mathbf{u}+\mathbf{v})-J(\mathbf{u}) \geq 0
$$

where we use the following notation:

$$
\begin{aligned}
a(\mathbf{u}, \mathbf{v}) & =2 \mu \int_{\Omega} D(\mathbf{u}): D(\mathbf{v}) d \mathbf{x}, \\
J(\mathbf{v}) & =\int_{\partial \Omega} g|\mathbf{v}| d \Gamma .
\end{aligned}
$$

We remark that relation (25) will be used as a first step to construct a numerical scheme (see Section 4 below).

Additionally, using the properties $\mathbf{u}_{n}=0$ and $\operatorname{div} \mathbf{u}=0$, we deduce that

$$
\int_{\Omega}\left(\frac{\partial \mathbf{u}}{\partial t}+(\mathbf{u} \cdot \nabla) \mathbf{u}\right) \cdot \mathbf{v} d \mathbf{x}=\frac{d}{d t} \int_{\Omega} \mathbf{u} \cdot \mathbf{v} d \mathbf{x}-\int_{\Omega}\left(\mathbf{u} \cdot \frac{\partial \mathbf{v}}{\partial t}+[(\mathbf{u} \cdot \nabla) \mathbf{v}] \cdot \mathbf{u}\right) d \mathbf{x} .
$$

Thus, replacing (28) in (24), integrating in time and taking $\mathbf{v}(T)=0$, the weak formulation writes as follows:

$$
\begin{aligned}
-\int_{\Omega} \mathbf{u}^{0}(\mathbf{x}) \cdot \mathbf{v}(0, \mathbf{x}) d \mathbf{x}-\int_{(0, T) \times \Omega} & \left(\mathbf{u} \cdot \frac{\partial \mathbf{v}}{\partial t}+[(\mathbf{u} \cdot \nabla) \mathbf{v}] \cdot \mathbf{u}\right) d \mathbf{x} d t \\
& +\int_{0}^{T} a(\mathbf{u}, \mathbf{v}) d t+\int_{0}^{T} J(\mathbf{u}+\mathbf{v}) d t-\int_{0}^{T} J(\mathbf{u}) d t \geq 0 .
\end{aligned}
$$

Definition 2.1. A weak solution $\mathbf{u}$ of the Navier-Stokes system with the Coulomb friction law (17)-(21) is a function

$$
\mathbf{u} \in L^{\infty}(0, T ; H) \cap L^{2}(0, T ; V)
$$

such that (29) holds true for all $\mathbf{v} \in \mathscr{C}_{c}^{1}([0, T) ; V)$.

We are now able to state our main result:

Theorem 2.2. If $\mathbf{u}^{0} \in H$, then there exists at least one weak solution of the Navier-Stokes system with the Coulomb friction law (17)-(21). Moreover, we have the following properties

$$
\begin{aligned}
\frac{\partial \mathbf{u}}{\partial t} \in L^{2}\left(0, T ; V^{\prime}\right) & \text { if } d=2, \\
\frac{\partial \mathbf{u}}{\partial t} \in L^{4 / 3}\left(0, T ; V^{\prime}\right) & \text { if } d=3,
\end{aligned}
$$

and for almost every $t \in[0, T]$, we have

$$
\frac{1}{2}\|\mathbf{u}(t)\|_{L^{2}(\Omega)^{d}}^{2}+\int_{0}^{t} a(\mathbf{u}, \mathbf{u}) d s+\int_{0}^{t} J(\mathbf{u}) d s \leq \frac{1}{2}\|\mathbf{u}(0)\|_{L^{2}(\Omega)^{d}}^{2} .
$$


Additionally, if $d=2$, we have in particular that the solution is unique and that

$$
\mathbf{u} \in \mathscr{C}^{0}([0, T] ; H)
$$

Remark 2.3. It is easy to prove that if $\mathbf{u}_{D}$ is a regular solution of the Navier-Stokes system with homogeneous Dirichlet boundary condition, then it satisfies the following well-known variational equation:

$$
\begin{aligned}
-\int_{\Omega} \mathbf{u}^{0}(\mathbf{x}) \cdot \mathbf{v}(0, \mathbf{x}) d \mathbf{x}- & \int_{(0, T) \times \Omega}\left(\mathbf{u}_{D} \cdot \frac{\partial \mathbf{v}}{\partial t}+\left[\left(\mathbf{u}_{D} \cdot \nabla\right) \mathbf{v}\right] \cdot \mathbf{u}_{D}\right) d \mathbf{x} d t \\
& +\int_{0}^{T} a\left(\mathbf{u}_{D}, \mathbf{v}\right) d t-\int_{(0, T) \times \partial \Omega}\left(\sigma\left(\mathbf{u}_{D}, p_{D}\right) \mathbf{n}\right)_{\tau} \cdot \mathbf{v} d \Gamma d t=0
\end{aligned}
$$

for all $\mathbf{v} \in \mathscr{C}_{c}^{1}([0, T) ; V)$. Then, if we compute the left hand side of (29), we get

$$
\int_{(0, T) \times \partial \Omega}\left(g|\mathbf{v}|+\left(\sigma\left(\mathbf{u}_{D}, p_{D}\right) \mathbf{n}\right)_{\tau} \cdot \mathbf{v}\right) d \Gamma d t
$$

from where we conclude that $\mathbf{u}_{D}$ is a solution of (29) if and only if $\left.\mid \sigma\left(\mathbf{u}_{D}, p_{D}\right) \mathbf{n}\right)_{\tau} \mid \leq g$.

\section{PROOF OF MAIN RESUlT}

For any $\varepsilon>0$ and $m \in \mathbb{N}^{*}$, we introduce a $(\varepsilon, m)$-regularized problem of (29) as follows: we begin by defining

$$
J_{\varepsilon}(\mathbf{v})=\int_{\partial \Omega} g j_{\varepsilon}(\mathbf{v}) d \Gamma
$$

where $j_{\varepsilon}(\mathbf{x})$ is a $\mathscr{C}^{1}$ convex regularized version of $|\mathbf{x}|$ satisfying the following properties:

$$
\begin{array}{rlrl}
j_{\varepsilon}(\mathbf{0}) & =0, & & \\
\nabla j_{\varepsilon}(\mathbf{x}) \cdot \mathbf{x} & \geq 0 & \forall \mathbf{x} \in \mathbb{R}^{d}, \\
\left|\nabla j_{\varepsilon}(\mathbf{x})\right| \leq 1 & \forall \mathbf{x} \in \mathbb{R}^{d}, \\
\left|j_{\varepsilon}(\mathbf{x})-\right| \mathbf{x}|| \leq \varepsilon & \forall \mathbf{x} \in \mathbb{R}^{d} .
\end{array}
$$

We then use the Galerkin method. To this end, let us consider an orthonormal basis $\left(\mathbf{v}_{j}\right)$ of $H$ such that $\mathbf{v}_{j} \in V$ and we denote by $V_{m}=\operatorname{Span}\left\{\mathbf{v}_{1}, \ldots, \mathbf{v}_{m}\right\}$. Then, we find the approximate solution of (29) as the function

$$
\mathbf{u}_{\varepsilon, m}(t, \mathbf{x})=\sum_{j=1}^{m} \varphi_{j}(t) \mathbf{v}_{j}(\mathbf{x}) \quad \text { with } \varphi_{j} \in \mathscr{C}^{1}(0, T),
$$

satisfying the following equation:

$$
\int_{\Omega} \frac{\partial \mathbf{u}_{\varepsilon, m}}{\partial t} \cdot \mathbf{v} d \mathbf{x}-\int_{\Omega}\left[\left(\mathbf{u}_{\varepsilon, m} \cdot \nabla\right) \mathbf{v}\right] \cdot \mathbf{u}_{\varepsilon, m} d \mathbf{x}+a\left(\mathbf{u}_{\varepsilon, m}, \mathbf{v}\right)+\int_{\partial \Omega} g \nabla j_{\varepsilon}\left(\mathbf{u}_{\varepsilon, m}\right) \cdot \mathbf{v} d \Gamma=0
$$

for any $\mathbf{v} \in V_{m}$, with the initial condition $\mathbf{u}_{\varepsilon, m}(0, \cdot)$ being the orthogonal projection of $\mathbf{u}^{0}$ onto $V_{m}$. We remark that in order to write (41) we have considered an approximation of inequality (25), where $|\mathbf{u}|$ has been approximated by the function $j_{\varepsilon}(\mathbf{u})$. Since this function is convex and differentiable, the variational inequality becomes a variational equation by using $\nabla j_{\varepsilon}$. 
By taking the test function $\mathbf{v}_{i}$, relation (41) can be written as

$$
\begin{aligned}
\varphi_{i}^{\prime}(t)-\sum_{j, k=1}^{m}\left[\int_{\Omega}\left[\left(\mathbf{v}_{j} \cdot \nabla\right) \mathbf{v}_{i}\right] \cdot \mathbf{v}_{k} d \mathbf{x}\right] \varphi_{j} \varphi_{k} & \left.+\sum_{j=1}^{m} a\left(\mathbf{v}_{j}, \mathbf{v}_{i}\right)\right] \varphi_{j} \\
& +\int_{\partial \Omega} g \nabla j_{\varepsilon}\left(\sum_{j=1}^{m} \varphi_{j}(t) \mathbf{v}_{j}(\mathbf{x})\right) \cdot \mathbf{v}_{i} d \Gamma=0,
\end{aligned}
$$

for all $i=1, \ldots, m$ and $\varphi_{i}(0)=\int_{\Omega} \mathbf{u}^{0} \cdot \mathbf{v}_{i} d \mathbf{x}$.

We note that equation (42) is of the form

$$
\varphi^{\prime}=F(\boldsymbol{\varphi}), \quad \boldsymbol{\varphi}(0)=\varphi^{0} \in \mathbb{R}^{m},
$$

with $F$ a Lipschitz continuous function (since $\varepsilon>0$ ). Thus, using the Cauchy-Lipschitz theorem, we deduce the existence of $a$ and then of $\mathbf{u}_{\varepsilon, m}$ which is a local solution of (41) for any $\mathbf{v} \in V_{m}$. Moreover, multiplying each equation of (42) by $\varphi_{i}$ and summing from $i=1$ to $m$, we deduce

$$
\frac{1}{2} \frac{d}{d t}\left\|\mathbf{u}_{\varepsilon, m}\right\|_{L^{2}(\Omega)^{d}}^{2}+a\left(\mathbf{u}_{\varepsilon, m}, \mathbf{u}_{\varepsilon, m}\right)+\int_{\partial \Omega} g \nabla j_{\varepsilon}\left(\mathbf{u}_{\varepsilon, m}\right) \cdot \mathbf{u}_{\varepsilon, m} d \Gamma=0 .
$$

This shows, due to (38), that $\mathbf{u}_{\varepsilon, m}$ is a global solution of (41) in $[0, T]$. Consequently, the sequence $\left(\mathbf{u}_{\varepsilon, m}\right)_{\varepsilon, m}$ is bounded in

$$
\mathscr{C}([0, T], H) \quad \text { and } \quad L^{\infty}(0, T ; H) \cap L^{2}(0, T ; V) .
$$

Moreover, using equation (41), we deduce that

$$
\left\|\frac{\partial \mathbf{u}_{\varepsilon, m}}{\partial t}(t)\right\|_{V^{\prime}} \leq C\left(\left\|\mathbf{u}_{\varepsilon, m}(t)\right\|_{L^{4}(\Omega)}^{2}+\left\|\mathbf{u}_{\varepsilon, m}(t)\right\|_{V}+1\right) .
$$

Then, using the classical Sobolev injections depending on the space dimension $d$ (see for instance [10, pp. 72-74]), we obtain that

$$
\begin{array}{r}
\left\|\frac{\partial \mathbf{u}_{\varepsilon, m}}{\partial t}(t)\right\|_{V^{\prime}} \leq C\left(\left\|\mathbf{u}_{\varepsilon, m}(t)\right\|_{H}\left\|\mathbf{u}_{\varepsilon, m}(t)\right\|_{V}+\left\|\mathbf{u}_{\varepsilon, m}(t)\right\|_{V}+1\right) \quad \text { if } d=2, \\
\left\|\frac{\partial \mathbf{u}_{\varepsilon, m}}{\partial t}(t)\right\|_{V^{\prime}} \leq C\left(\left\|\mathbf{u}_{\varepsilon, m}(t)\right\|_{H}^{1 / 2}\left\|\mathbf{u}_{\varepsilon, m}(t)\right\|_{V}^{3 / 2}+\left\|\mathbf{u}_{\varepsilon, m}(t)\right\|_{V}+1\right) \quad \text { if } d=3 .
\end{array}
$$

From the above estimates and from the regularities (44) of $\mathbf{u}_{\varepsilon, m}$, we also deduce that the sequence $\left(\frac{\partial \mathbf{u}_{\varepsilon, m}}{\partial t}\right)_{\varepsilon, m}$ is bounded in

$$
\begin{aligned}
L^{2}\left(0, T ; V^{\prime}\right) & \text { if } d=2, \\
L^{4 / 3}\left(0, T ; V^{\prime}\right) & \text { if } d=3 .
\end{aligned}
$$

Consequently, taking $\varepsilon=\frac{1}{m}$, and passing to the limit as $m \rightarrow \infty$, we get that, up to a subsequence,

$$
\begin{aligned}
\mathbf{u}_{\varepsilon, m} \rightarrow \mathbf{u} & \text { weakly* in } L^{\infty}(0, T ; H) \cap L^{2}(0, T ; V), \\
\frac{\partial \mathbf{u}_{\varepsilon, m}}{\partial t} \rightarrow \frac{\partial \mathbf{u}}{\partial t} & \text { weakly in } L^{2}\left(0, T ; V^{\prime}\right) \text { if } d=2, \\
\frac{\partial \mathbf{u}_{\varepsilon, m}}{\partial t} \rightarrow \frac{\partial \mathbf{u}}{\partial t} & \text { weakly in } L^{4 / 3}\left(0, T ; V^{\prime}\right) \text { if } d=3 .
\end{aligned}
$$


In order to pass to the limit in the nonlinear terms, we use the compactness result stated in Theorem 2.1 from [17, pp. 271]. To this end, we define the space $V^{\eta}$ as follows

$$
V^{\eta}=\left\{\mathbf{v} \in H^{\eta}(\Omega)^{d}: \operatorname{div} \mathbf{v}=0, \mathbf{v}_{n}=0 \text { on } \partial \Omega\right\},
$$

with $H^{\eta}(\Omega)$ the classical interpolated Sobolev space (for its definition see, for instance, [11, Chapter 9]. Since, for any $\eta \in(0,1), V \subset V^{\eta} \subset H$, where the first injection is compact (see [11, Theorem 16.1]) then, from Theorem 2.1 in [17, pp. 271], we obtain that

$$
\mathbf{u}_{\varepsilon, m} \rightarrow \mathbf{u} \quad \text { strongly in } L^{2}\left(0, T ; V^{\eta}\right),
$$

and, in particular, for $\eta>1 / 2$, we deduce that

$$
\mathbf{u}_{\varepsilon, m} \rightarrow \mathbf{u} \quad \text { strongly in } L^{2}\left(0, T ; L^{2}(\partial \Omega)\right) .
$$

In order to pass to the limit, we begin by rewriting (41), for any $\mathbf{v} \in \mathscr{C}_{c}^{1}\left([0, T) ; V_{m}\right)$ and integrating over $[0, T]$. We have

$$
\begin{aligned}
-\int_{\Omega} \mathbf{u}_{\varepsilon, m}^{0}(\mathbf{x}) \cdot \mathbf{v}(0, \mathbf{x}) d \mathbf{x}- & \int_{(0, T) \times \Omega}\left(\mathbf{u}_{\varepsilon, m} \cdot \frac{\partial \mathbf{v}}{\partial t}+\left[\left(\mathbf{u}_{\varepsilon, m} \cdot \nabla\right) \mathbf{v}\right] \cdot \mathbf{u}_{\varepsilon, m}\right) d \mathbf{x} d t \\
& +\int_{0}^{T} a\left(\mathbf{u}_{\varepsilon, m}, \mathbf{v}\right) d t+\int_{(0, T) \times \partial \Omega} g \nabla j_{\varepsilon}\left(\mathbf{u}_{\varepsilon, m}\right) \cdot \mathbf{v} d \Gamma d t=0 .
\end{aligned}
$$

Since $j_{\varepsilon}$ is a convex function, we have

$$
\nabla j_{\varepsilon}\left(\mathbf{u}_{\varepsilon, m}\right) \cdot\left(\mathbf{v}+\mathbf{u}_{\varepsilon, m}-\mathbf{u}_{\varepsilon, m}\right) \leq j_{\varepsilon}\left(\mathbf{v}+\mathbf{u}_{\varepsilon, m}\right)-j_{\varepsilon}\left(\mathbf{u}_{\varepsilon, m}\right)
$$

Using the above inequality in (54), it implies that for any $\mathbf{v} \in \mathscr{C}_{c}^{1}\left([0, T) ; V_{m}\right)$,

$$
\begin{aligned}
-\int_{\Omega} \mathbf{u}_{\varepsilon, m}^{0}(\mathbf{x}) \cdot \mathbf{v}(0, \mathbf{x}) d \mathbf{x}-\int_{(0, T) \times \Omega}\left(\mathbf{u}_{\varepsilon, m} \cdot \frac{\partial \mathbf{v}}{\partial t}+\left[\left(\mathbf{u}_{\varepsilon, m} \cdot \nabla\right) \mathbf{v}\right] \cdot \mathbf{u}_{\varepsilon, m}\right) d \mathbf{x} d t \\
+\int_{0}^{T} a\left(\mathbf{u}_{\varepsilon, m}, \mathbf{v}\right) d t+\int_{0}^{T} J_{\varepsilon}\left(\mathbf{v}+\mathbf{u}_{\varepsilon, m}\right) d t-\int_{0}^{T} J_{\varepsilon}\left(\mathbf{u}_{\varepsilon, m}\right) d t \geq 0 .
\end{aligned}
$$

Let fix $\mathbf{v} \in \mathscr{C}_{c}^{1}\left([0, T) ; V_{m_{0}}\right)$ and let pass to the limit in (56). From the weak convergence (49)), it follows that we can pass to the limit in all linear terms. Moreover, using the compactness properties (52) -(53) and property (40), we can also pass to the limit in the nonlinear terms and thus we get

$$
\begin{aligned}
-\int_{\Omega} \mathbf{u}^{0}(\mathbf{x}) \cdot \mathbf{v}(0, \mathbf{x}) d \mathbf{x}-\int_{(0, T) \times \Omega} & \left(\mathbf{u} \cdot \frac{\partial \mathbf{v}}{\partial t}+[(\mathbf{u} \cdot \nabla) \mathbf{v}] \cdot \mathbf{u}\right) d \mathbf{x} d t \\
& +\int_{0}^{T} a(\mathbf{u}, \mathbf{v}) d t+\int_{0}^{T} J(\mathbf{v}+\mathbf{u}) d t-\int_{0}^{T} J(\mathbf{u}) d t \geq 0
\end{aligned}
$$

for any $\mathbf{v} \in \mathscr{C}_{c}^{1}\left([0, T) ; V_{m_{0}}\right)$.

For any $\mathbf{v} \in \mathscr{C}_{c}^{1}([0, T) ; V)$, we denote by $P_{m} \mathbf{v}$ the orthogonal projection of $\mathbf{v}$ on $V_{m}$ with respect to the inner product on $V$. We use $P_{m} \mathbf{v}$ as the test function in (57) and we get

$$
\begin{aligned}
-\int_{\Omega} \mathbf{u}^{0}(\mathbf{x}) \cdot P_{m} \mathbf{v}(0, \mathbf{x}) & d \mathbf{x}-\int_{(0, T) \times \Omega}\left(\mathbf{u} \cdot \frac{\partial P_{m} \mathbf{v}}{\partial t}+\left[(\mathbf{u} \cdot \nabla) P_{m} \mathbf{v}\right] \cdot \mathbf{u}\right) d \mathbf{x} d t \\
& +\int_{0}^{T} a\left(\mathbf{u}, P_{m} \mathbf{v}\right) d t+\int_{0}^{T} J\left(P_{m} \mathbf{v}+\mathbf{u}\right) d t-\int_{0}^{T} J(\mathbf{u}) d t \geq 0 .
\end{aligned}
$$

Therefore, using the strong convergence of $P_{m} \mathbf{v}$ to $\mathbf{v}$ in $\mathscr{C}^{1}([0, T] ; V)$, we can pass to the limit as $m \rightarrow \infty$ in all terms of (58) and we get the inequality (29) for all $\mathbf{v} \in \mathscr{C}_{c}^{1}([0, T) ; V)$. 
Therefore, we have proved the existence of a weak solution of the Navier-Stokes system with the Coulomb friction law stated in Theorem 2.2 .

Let us now prove the inequality (32). To this end, we integrate (43) in $[0, t]$ and we get

$$
\begin{aligned}
\frac{1}{2}\left\|\mathbf{u}_{\varepsilon, m}(t)\right\|_{L^{2}(\Omega)^{d}}^{2}-\frac{1}{2}\left\|\mathbf{u}_{\varepsilon, m}(0)\right\|_{L^{2}(\Omega)^{d}}^{2} & +\int_{0}^{t} a\left(\mathbf{u}_{\varepsilon, m}, \mathbf{u}_{\varepsilon, m}\right) d s \\
& \quad+\int_{0}^{t} \int_{\partial \Omega} g \nabla j_{\varepsilon}\left(\mathbf{u}_{\varepsilon, m}\right) \cdot \mathbf{u}_{\varepsilon, m} d \Gamma d s=0 .
\end{aligned}
$$

Since $j_{\varepsilon}$ is a convex function, we have $j_{\varepsilon}(\mathbf{0}) \geq j_{\varepsilon}\left(\mathbf{u}_{\varepsilon, m}\right)+\nabla j_{\varepsilon}\left(\mathbf{u}_{\varepsilon, m}\right) \cdot\left(\mathbf{0}-\mathbf{u}_{\varepsilon, m}\right)$ and using (37), we can write

$$
\frac{1}{2}\left\|\mathbf{u}_{\varepsilon, m}(t)\right\|_{L^{2}(\Omega)^{d}}^{2}-\frac{1}{2}\left\|\mathbf{u}_{\varepsilon, m}(0)\right\|_{L^{2}(\Omega)^{d}}^{2}+\int_{0}^{t} a\left(\mathbf{u}_{\varepsilon, m}, \mathbf{u}_{\varepsilon, m}\right) d s+\int_{0}^{t} J_{\varepsilon}\left(\mathbf{u}_{\varepsilon, m}\right) d s \leq 0 .
$$

We multiply this equality by $\phi(t)$, where $\phi \in \mathscr{D}((0, T)), \phi(t) \geq 0$, and we integrate in time to get

$$
\int_{0}^{T} \phi(t)\left\{\frac{1}{2}\left\|\mathbf{u}_{\varepsilon, m}(t)\right\|_{L^{2}(\Omega)^{d}}^{2}-\frac{1}{2}\left\|\mathbf{u}_{\varepsilon, m}(0)\right\|_{L^{2}(\Omega)^{d}}^{2}+\int_{0}^{t} a\left(\mathbf{u}_{\varepsilon, m}, \mathbf{u}_{\varepsilon, m}\right) d s+\int_{0}^{t} J_{\varepsilon}\left(\mathbf{u}_{\varepsilon, m}\right) d s\right\} d t \leq 0 .
$$

Then, by taking the limit inferior of the above inequality and using the convergences (49) and (53), we obtain

$$
\int_{0}^{T} \phi(t)\left\{\frac{1}{2}\|\mathbf{u}(t)\|_{L^{2}(\Omega)^{d}}^{2}-\frac{1}{2}\|\mathbf{u}(0)\|_{L^{2}(\Omega)^{d}}^{2}+\int_{0}^{t} a(\mathbf{u}, \mathbf{u}) d s+\int_{0}^{t} J(\mathbf{u}) d s\right\} d t \leq 0 .
$$

Thus, inequality (32) is a direct consequence of the fact that the above estimate is valid for any $\phi \in \mathscr{D}((0, T)), \phi(t) \geq 0$.

Finally, let us prove the uniqueness in the 2 -dimensional case $(d=2)$. First, from Lemma 1.2 in [17, p. 260], we obtain (33). We can integrate by parts in (29) and rewrite it as

$$
\begin{aligned}
\int_{(0, T)}\left\langle\frac{\partial \mathbf{u}}{\partial t}, \mathbf{v}\right\rangle_{V^{\prime}, V} d t & -\int_{(0, T) \times \Omega} \\
& {[(\mathbf{u} \cdot \nabla) \mathbf{v}] \cdot \mathbf{u} d \mathbf{x} d t } \\
& +\int_{0}^{T} a(\mathbf{u}, \mathbf{v}) d t+\int_{0}^{T} J(\mathbf{u}+\mathbf{v}) d t-\int_{0}^{T} J(\mathbf{u}) d t \geq 0 .
\end{aligned}
$$

Now, a density argument shows that (63) holds for test functions $\mathbf{v}$ such that

$$
\mathbf{v} \in L^{2}(0, T ; V)
$$

In particular, for any $\mathbf{w} \in L^{2}(0, T ; V)$ and any $t \in[0, T]$, we can take the special test function $\mathbf{v}$ defined by

$$
\mathbf{v}(s)= \begin{cases}\mathbf{w}(s)-\mathbf{u}(s) & \text { if } s \in[0, t] \\ \mathbf{0} & \text { if } s \in(t, T]\end{cases}
$$


and we obtain:

$$
\begin{aligned}
\int_{(0, t)}\left\langle\frac{\partial \mathbf{u}}{\partial t}, \mathbf{w}-\mathbf{u}\right\rangle_{V^{\prime}, V} d s- & \int_{(0, t) \times \Omega}[(\mathbf{u} \cdot \nabla) \mathbf{w}] \cdot \mathbf{u} d \mathbf{x} d s \\
& +\int_{0}^{t} a(\mathbf{u}, \mathbf{w}-\mathbf{u}) d s+\int_{0}^{t} J(\mathbf{w}) d s-\int_{0}^{t} J(\mathbf{u}) d s \geq 0 .
\end{aligned}
$$

Assume now that we have two solutions $\mathbf{u}^{(1)}$ and $\mathbf{u}^{(2)}$ of system (65). Taking $\mathbf{w}=\mathbf{u}^{(2)}$ as a test function in the inequality (65) satisfied by $\mathbf{u}^{(1)}$ and taking $\mathbf{w}=\mathbf{u}^{(1)}$ as a test function in the inequality (65) satisfied by $\mathbf{u}^{(2)}$, we deduce

$$
\begin{aligned}
& \frac{1}{2} \int_{(0, t)} \frac{\partial}{\partial t}\left\|\mathbf{u}^{(1)}-\mathbf{u}^{(2)}\right\|_{L^{2}(\Omega)^{2}}^{2} d s+2 \mu \int_{(0, t) \times \Omega}\left|D\left(\mathbf{u}^{(2)}-\mathbf{u}^{(1)}\right)\right|^{2} d \mathbf{x} d s \\
& \quad \leq \int_{(0, t) \times \Omega}\left[\left(\mathbf{u}^{(1)} \cdot \nabla\right) \mathbf{u}^{(1)}\right] \cdot \mathbf{u}^{(2)} d \mathbf{x} d s-\int_{(0, t) \times \Omega}\left[\left(\mathbf{u}^{(2)} \cdot \nabla\right) \mathbf{u}^{(1)}\right] \cdot \mathbf{u}^{(2)} d \mathbf{x} d s \\
& \quad=-\int_{(0, t) \times \Omega}\left[\left(\left(\mathbf{u}^{(1)}-\mathbf{u}^{(2)}\right) \cdot \nabla\right) \mathbf{u}^{(1)}\right] \cdot\left(\mathbf{u}^{(1)}-\mathbf{u}^{(2)}\right) d \mathbf{x} d s .
\end{aligned}
$$

The above inequality and the Sobolev embedding theorem yield

$$
\begin{aligned}
& \frac{1}{2}\left\|\mathbf{u}^{(1)}(t)-\mathbf{u}^{(2)}(t)\right\|_{L^{2}(\Omega)^{2}}^{2}+2 \mu \int_{(0, t) \times \Omega}\left|D\left(\mathbf{u}^{(2)}-\mathbf{u}^{(1)}\right)\right|^{2} d \mathbf{x} d s \\
& \quad \leq C \int_{(0, t)}\left\|\mathbf{u}^{(1)}-\mathbf{u}^{(2)}\right\|_{H^{1}(\Omega)^{2}}\left\|\mathbf{u}^{(1)}-\mathbf{u}^{(2)}\right\|_{L^{2}(\Omega)^{2}}\left\|\mathbf{u}^{(1)}\right\|_{H^{1}(\Omega)^{2}} d s .
\end{aligned}
$$

By using the Young, PoincarẪl' and Korn inequalities we get

$$
\frac{1}{2}\left\|\mathbf{u}^{(1)}(t)-\mathbf{u}^{(2)}(t)\right\|_{L^{2}(\Omega)^{2}}^{2} \leq C \int_{(0, t)}\left\|\mathbf{u}^{(1)}-\mathbf{u}^{(2)}\right\|_{L^{2}(\Omega)^{2}}^{2}\left\|\mathbf{u}^{(1)}\right\|_{H^{1}(\Omega)^{2}}^{2} d s .
$$

Uniqueness is a direct consequence of the Gronwall inequality applied to (68) and thus we conclude the proof of Theorem 2.2 .

\section{Numerical Tests}

In order to write a numerical algorithm to solve the Navier-Stokes/Coulomb friction law system (17)-(21), we begin by writing a mixed formulation of inequality (25). To this end, we introduce the following vectorial spaces

$$
\begin{aligned}
& M=\left\{q \in L^{2}(\Omega): \int_{\Omega} q d \mathbf{x}=0\right\}, \\
& V_{0}=\left\{\mathbf{v} \in H^{1}(\Omega)^{d}: \mathbf{v}_{n}=0 \text { on } \partial \Omega\right\} .
\end{aligned}
$$

Using these spaces, the problem (17)-(21) can be written as follows: Find $(\mathbf{u}, p) \in V_{0} \times M$ such that

$$
\begin{gathered}
\int_{\Omega}\left(\frac{\partial \mathbf{u}}{\partial t}+(\mathbf{u} \cdot \nabla) \mathbf{u}\right) \cdot \mathbf{v} d \mathbf{x}+a(\mathbf{u}, \mathbf{v})+b(\mathbf{v}, p) \geq J(\mathbf{u})-J(\mathbf{u}+\mathbf{v}) \quad \forall \mathbf{v} \in V_{0} \\
b(\mathbf{u}, q)=0 \quad \forall q \in M,
\end{gathered}
$$

for a.e. $t \in(0, T)$, where

$$
b(\mathbf{u}, q)=-\int_{\Omega} \operatorname{div} \mathbf{u} q d \mathbf{x} \quad \forall \mathbf{u} \in V_{0}, \quad q \in M .
$$


It is easy to prove that the system (69)-(70) is equivalent to equation (25).

In order to treat the nonlinear term $(\mathbf{u} \cdot \nabla) \mathbf{u}$, we use the characteristic functions method (see, for instance, [12, 16, 14, 13]). More precisely, we define the characteristic function $\psi:[0, T]^{2} \times \Omega \rightarrow \Omega$ as the solution of the initial value problem

$$
\left\{\begin{array}{l}
\frac{d}{d t} \boldsymbol{\psi}(t ; s, \mathbf{x})=\mathbf{u}(t, \boldsymbol{\psi}(t ; s, \mathbf{x})) \quad \forall t \in[0, T] \\
\boldsymbol{\psi}(s ; s, \mathbf{x})=\mathbf{x}
\end{array}\right.
$$

It is well-known that the material derivative $\frac{d \mathbf{u}}{d t}=\frac{\partial \mathbf{u}}{\partial t}+(\mathbf{u} \cdot \nabla) \mathbf{u}$ of $\mathbf{u}$ at instant $t_{0}$ satisfies:

$$
\frac{d \mathbf{u}}{d t}\left(t_{0}, \mathbf{x}\right)=\frac{\partial}{\partial t}\left[\mathbf{u}\left(t, \boldsymbol{\psi}\left(t ; t_{0}, \mathbf{x}\right)\right)\right]_{\left.\right|_{t=t_{0}}} .
$$

Additionally, we introduce two families of finite element spaces which approximate the spaces $V_{0}$ and $M$. To this end, we consider the discretization parameter $h>0$ and a quasiuniform triangulation $\mathcal{T}_{h}$ of the domain $\Omega$. We denote by $\mathcal{W}_{h}$ the $\mathbb{P}_{2}$-finite element space associated with $\mathcal{T}_{h}$ for the velocity field in the Stokes problem and by $M_{h}$ the $\mathbb{P}_{1}$-finite element space for the pressure (see for instance [6]). Then, we define the following finite element space for a conform approximation:

$$
V_{h}=\mathcal{W}_{h} \cap V_{0}
$$

Finally, to approximate the functional $J(\mathbf{v})$, we use the function $J_{h}(\mathbf{v})$ defined by:

$$
J_{h}(\mathbf{v})=\int_{\partial \Omega} g j_{h}(\mathbf{v}) d \Gamma
$$

where

$$
j_{h}(\mathbf{v})= \begin{cases}\frac{1}{4 h}|\mathbf{v}|^{2} & \text { if }|\mathbf{v}|<2 h \\ |\mathbf{v}|-h & \text { if }|\mathbf{v}| \geq 2 h\end{cases}
$$

Using this notation, the discretization of our problem is the following:

Let $N$ be a positive integer. We denote $\Delta t=T / N$ and $t_{k}=k \Delta t$ for all $k \in\{0, \ldots, N\}$. Assume that the approximate solution $\left(\mathbf{u}_{h}^{k}, p_{h}^{k}\right)$ of (69)-(70) at $t=t_{k}$ is known. We describe below the numerical scheme allowing to determinate the approximate solution $\left(\mathbf{u}_{h}^{k+1}, p_{h}^{k+1}\right)$ at time $t=t_{k+1}$. First, we compute the approximated characteristic function $\boldsymbol{\psi}_{h}^{k}$ defined as the solution of

$$
\left\{\begin{array}{l}
\frac{d}{d t} \boldsymbol{\psi}_{h}^{k}\left(t ; t_{k+1}, \mathbf{x}\right)=\mathbf{u}_{h}^{k}\left(\boldsymbol{\psi}_{h}^{k}\left(t ; t_{k+1}, \mathbf{x}\right)\right) \quad \forall t \in\left[t_{k}, t_{k+1}\right] \\
\boldsymbol{\psi}_{h}^{k}\left(t_{k+1} ; t_{k+1}, \mathbf{x}\right)=\mathbf{x} .
\end{array}\right.
$$

Then, we define

$$
\overline{\mathbf{X}}_{h}^{k}(\mathbf{x})=\boldsymbol{\psi}_{h}^{k}\left(t_{k} ; t_{k+1}, \mathbf{x}\right) \quad \forall \mathbf{x} \in \Omega .
$$

With these notations, we consider the following mixed variational fully discrete formulation: Find $\left(\mathbf{u}_{h}^{k+1}, p_{h}^{k+1}\right) \in V_{h} \times M_{h}$ such that

$$
\int_{\Omega}\left(\frac{\mathbf{u}_{h}^{k+1}-\mathbf{u}_{h}^{k} \circ \overline{\mathbf{X}}_{h}^{k}}{\Delta t}\right) \cdot \mathbf{v} d \mathbf{x}+a\left(\mathbf{u}_{h}^{k+1}, \mathbf{v}\right)+b\left(\mathbf{v}, p_{h}^{k+1}\right)+\int_{\partial \Omega} g \nabla j_{h}\left(\mathbf{u}_{h}^{k+1}\right) \cdot \mathbf{v} d \Gamma=0 \quad \forall \mathbf{v} \in V_{h},
$$

$$
b\left(\mathbf{u}_{h}^{k+1}, q\right)=0 \quad \forall q \in M_{h} .
$$


We remark that since the approximation $j_{h}(\mathbf{v})$ of $|\mathbf{u}|$ is convex and differentiable, the discrete formulation associated with the variational inequality (69) is a variational equation by using $\nabla j_{h}$. It is clear from (75) that

$$
\nabla j_{h}(\mathbf{v})=\frac{1}{\max \{2 h,|\mathbf{v}|\}} \mathbf{v} .
$$

Consequently, the discrete formulation writes: Find $\left(\mathbf{u}_{h}^{k+1}, p_{h}^{k+1}\right) \in V_{h} \times M_{h}$ such that

$$
\begin{aligned}
& \int_{\Omega}\left(\frac{\mathbf{u}_{h}^{k+1}-\mathbf{u}_{h}^{k} \circ \overline{\mathbf{X}}_{h}^{k}}{\Delta t}\right) \cdot \mathbf{v} \quad d \mathbf{x}+ a\left(\mathbf{u}_{h}^{k+1}, \mathbf{v}\right)+b\left(\mathbf{v}, p_{h}^{k+1}\right) \\
&+\int_{\partial \Omega} \frac{g}{\max \left\{2 h,\left|\mathbf{u}_{h}^{k+1}\right|\right\}} \mathbf{u}_{h}^{k+1} \cdot \mathbf{v} d \Gamma=0 \quad \forall \mathbf{v} \in V_{h}, \\
& b\left(\mathbf{u}_{h}^{k+1}, q\right)=0 \quad \forall q \in M_{h} .
\end{aligned}
$$

The discretized system (81)-(82) is still nonlinear, due to the boundary integral. In order to deal with this nonlinearity, we use an iterative fixed point method, where we compute $\mathbf{u}_{h}^{k+1, i+1}$ in term of $\mathbf{u}_{h}^{k+1, i}$ by solving (81)-(82), with the boundary integral replaced by

$$
\int_{\partial \Omega} \frac{g}{\max \left\{2 h,\left|\mathbf{u}_{h}^{k+1, i}\right|\right\}} \mathbf{u}_{h}^{k+1, i+1} \cdot \mathbf{v} d \Gamma .
$$

Let us now use the numerical scheme defined in (81)- (82) in two numerical tests. In the first one, we study the flow of a viscous incompressible fluid through the rectangular channel with an abrupt contraction (see Figure 1 below). In the second example, we consider the classical fluid flow after a cylindrical obstacle in a rectangular channel (see Figure 2 below).

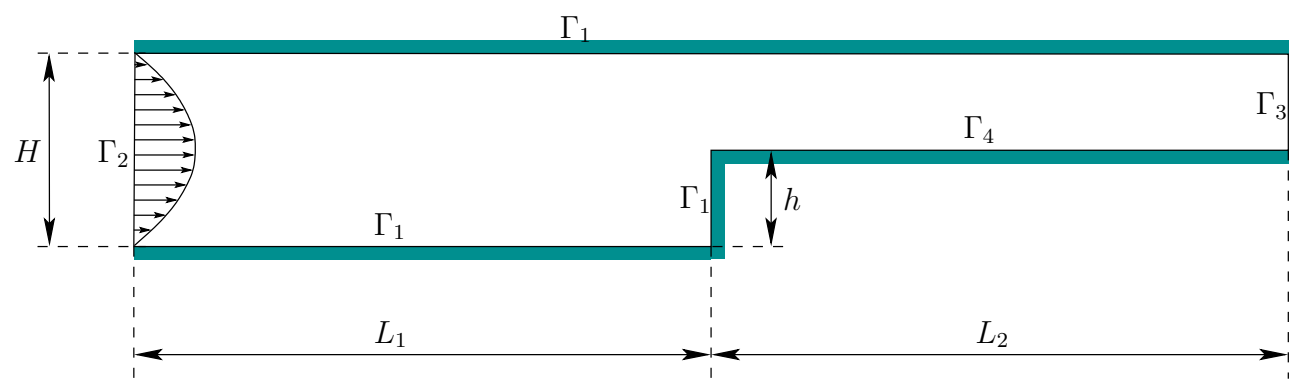

FiguRE 1. Horizontal channel with an abrupt contraction.

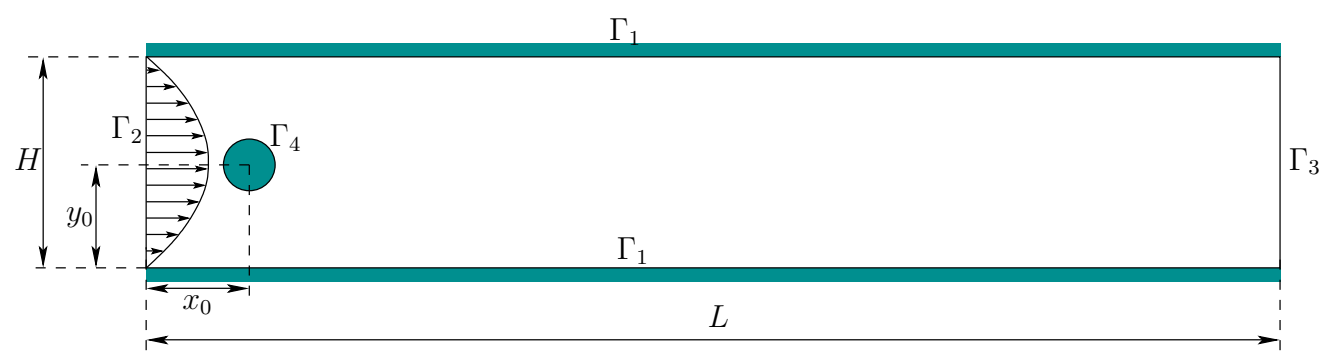

FiguRE 2. Horizontal channel with a cylindrical obstacle.

In both of these domains, we decompose the domain boundary in subsets where we impose the following different boundary conditions: 
$\Gamma_{1}$ : Homogeneous Dirichlet boundary condition $\left(u_{x}=u_{y}=0\right)$, modelling the contact with an infinitely adherent wall.

$\Gamma_{2}$ : Inlet Dirichlet boundary condition, where the inlet velocity field is given by a parabolic profile with a maximum value equal to $u_{\max }\left(u_{x}=4 u_{\max } \frac{y}{H}\left(1-\frac{y}{H}\right), u_{y}=0\right)$.

$\Gamma_{3}$ : Outlet boundary condition $\left((\sigma n)_{n}=0, u_{y}=0\right)$.

$\Gamma_{4}$ : Special wall where we study the Coulomb law effect.

For each example, we are interested in the numerical influence of the Coulomb law on $\Gamma_{4}$ over the solution of Navier-Stokes system. For this reason, we compare the solution obtained using this new boundary condition with the solutions resulted by imposing the classical Dirichlet and Neumann boundary conditions on $\Gamma_{4}$. To this end, we present three simulations considering the following boundary conditions on $\Gamma_{4}$ :

a) Homogeneous Dirichlet boundary condition for both components of the velocity field $\left(\mathbf{u}_{n}=0\right.$ and $\left.\mathbf{u}_{\tau}=0\right)$.

b) Homogeneous Dirichlet boundary condition for the normal velocity field and Neumann boundary condition for the tangential component of the stress field $\left(\mathbf{u}_{n}=0\right.$ and $\left.(\sigma \mathbf{n})_{\tau}=0\right)$.

c) Coulomb boundary condition given in (6) $-(8)$.

The simulations considered are divided in two parts. In the first one, in order to construct an initial velocity field, we solve the Stokes system considering one of the three above boundary conditions on $\Gamma_{4}$. Then, using this initial condition, we solve Navier-Stokes system for $t \in[0, T]$ with the previous corresponding conditions on $\Gamma_{4}$. In the sequel, we show the most relevant results of these computations for both domains, separately.

The simulations presented here were partially made with the software FreeFem++ [8] (for the case of the Dirichlet boundary condition and the Neumann boundary condition) and with the software Matlab (for the Coulomb law).

4.1. Results for the rectangular channel with an abrupt contraction. In this section, we show the numerical results obtained for the solutions of Stokes and Navier-Stokes systems for the first geometry given in Figure 1. In the case of the Coulomb law boundary condition, we consider the parameter $g=0.015$, because it is smaller than the tangential stress obtained by imposing Dirichlet boundary condition. We have verified that if this parameter is greater than the tangential stress of the problem with the boundary condition a), the solutions of Navier-Stokes system with Coulomb and Dirichlet boundary conditions coincide.

In Figure 3, we present the velocities fields obtained as solution of the Stokes system for the three considered boundary conditions. These velocities fields are considered to be the initial conditions for the Navier-Stokes system. In Figures 45, we see the corresponding velocities fields obtained solving Navier-Stokes system at time $t=1 \mathrm{~s}$ and $t=5 \mathrm{~s}$. In Figures 6 9, we see the corresponding tangential stress $(\sigma \mathbf{n})_{\tau}$ and tangential velocities fields $\mathbf{u}_{\tau}$ obtained solving Navier-Stokes system at time $t=1 \mathrm{~s}$ and $t=5 \mathrm{~s}$. 

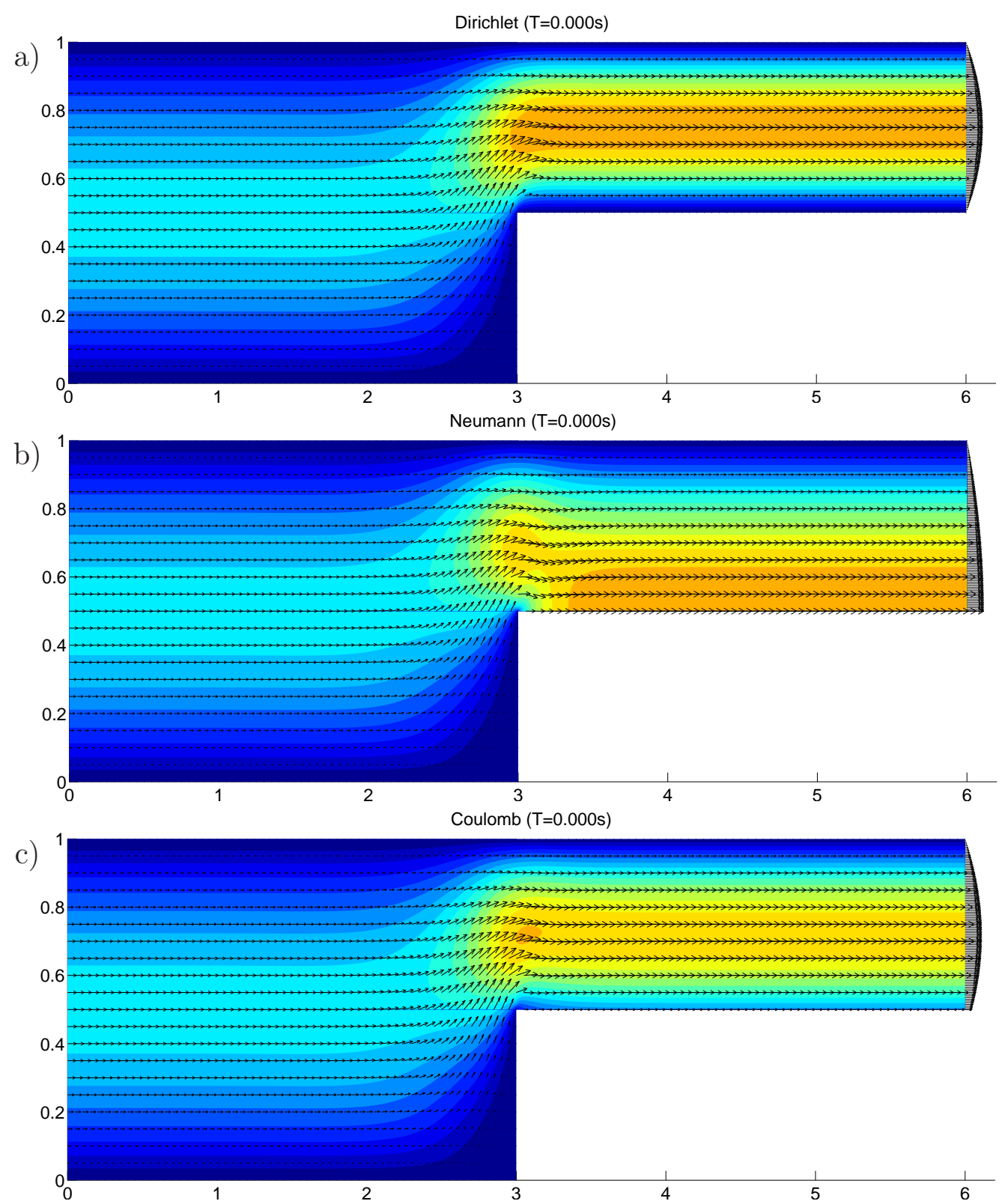

FiguRE 3. Velocity field at $t=0$, obtained as the solution of the Stokes system with the following boundary conditions on $\Gamma_{4}$ : a) Homogeneous Dirichlet boundary condition; b) Neumann boundary condition; c) Coulomb boundary condition. 

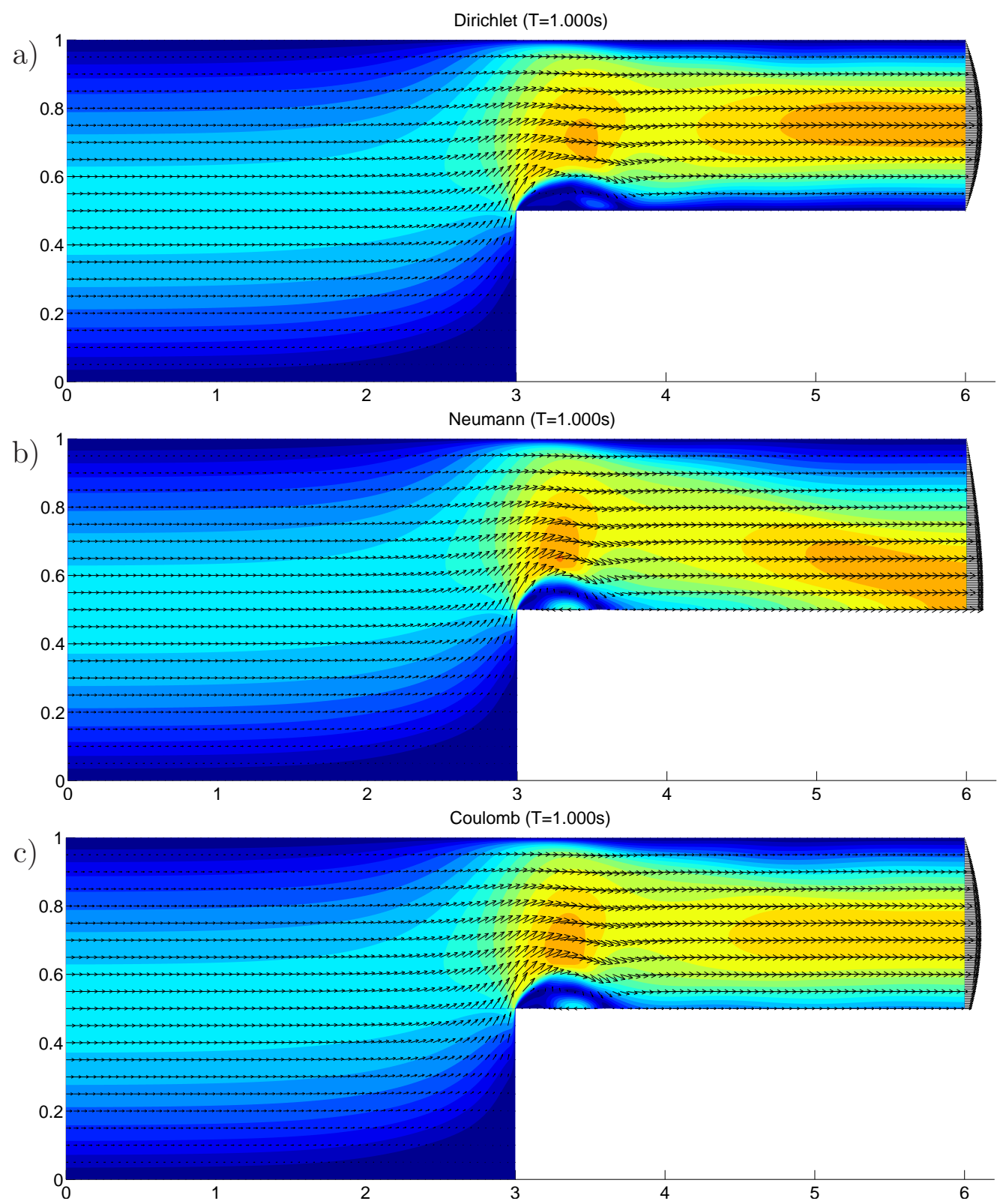

FiguRE 4. Velocity field at $t=1 s$, obtained as the solution of Navier-Stokes equation with the three boundary conditions on $\Gamma_{4}$ : a) Homogeneous Dirichlet boundary condition; b) Neumann boundary condition; c) Coulomb boundary condition. 


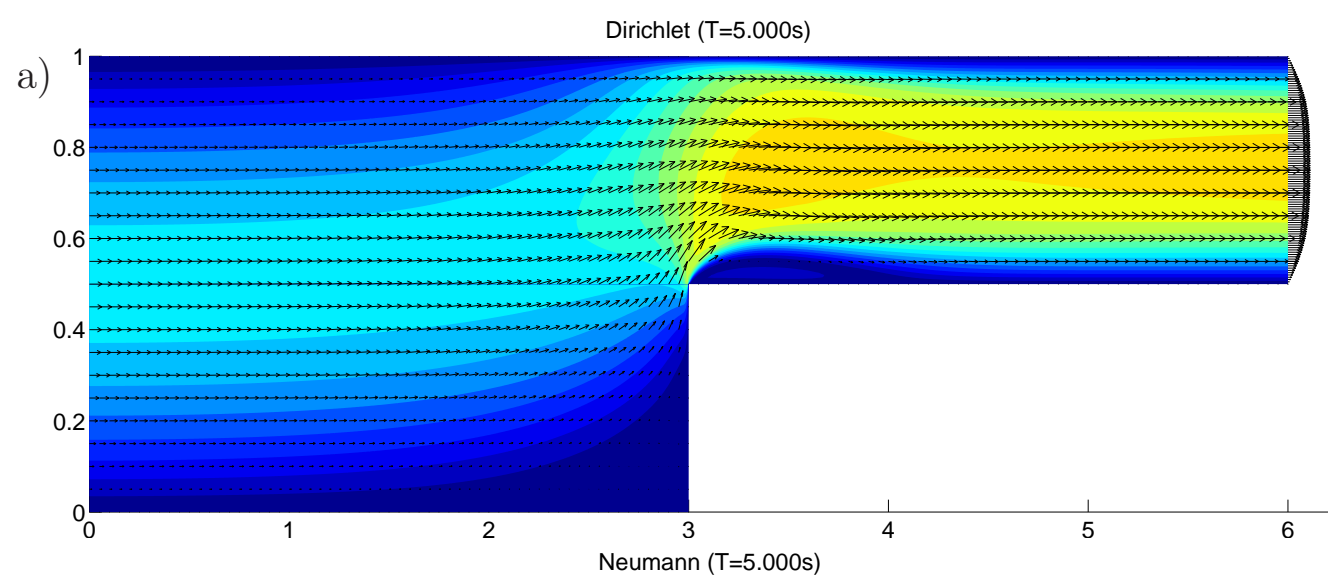

b)
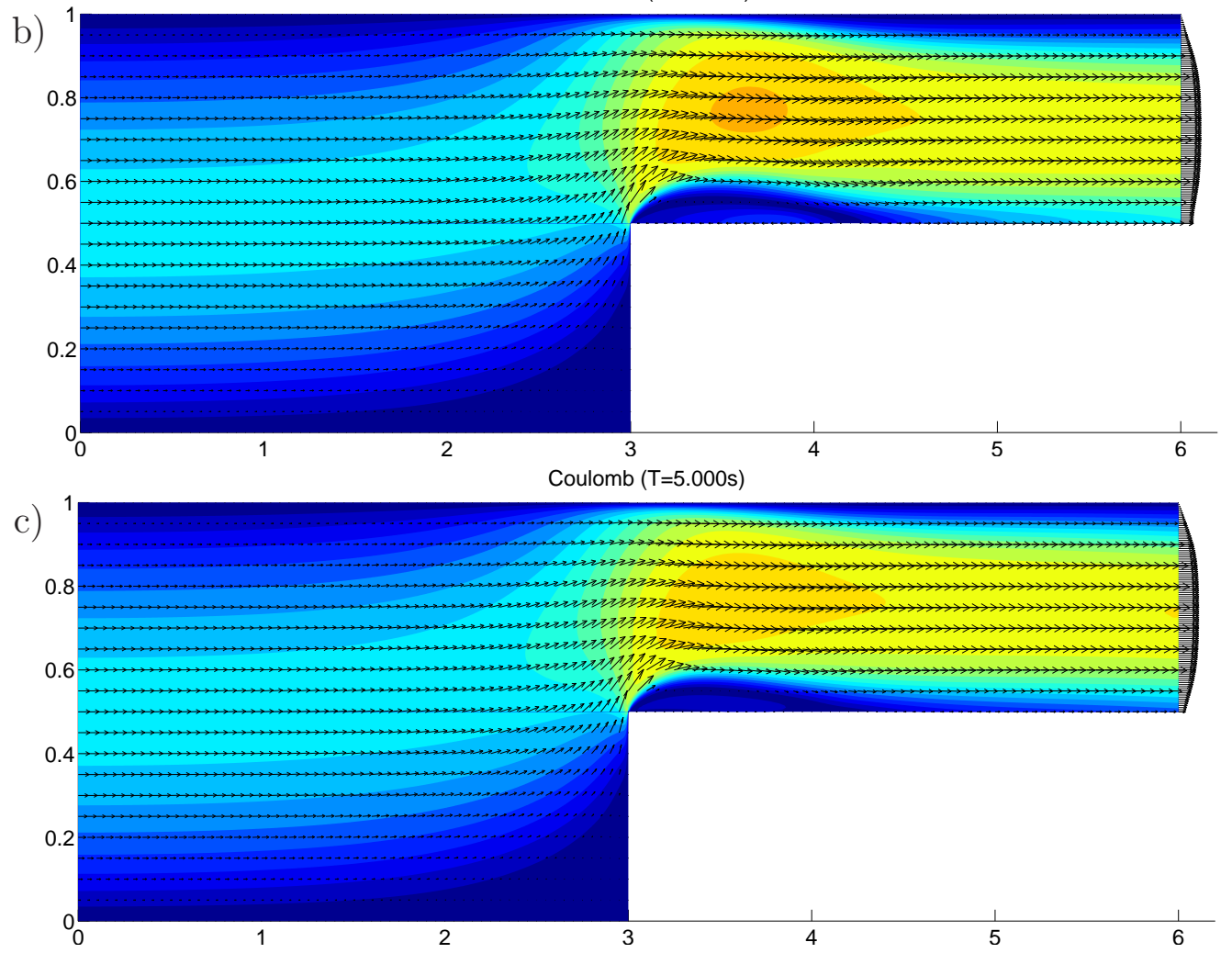

FiguRE 5. Velocity field at $t=5 \mathrm{~s}$, obtained as the solution of Navier-Stokes equation with the three boundary conditions on $\Gamma_{4}$ : a) Homogeneous Dirichlet boundary condition; b) Neumann boundary condition; c) Coulomb boundary condition. 
In Figure 6, we represent the tangential stress $(\sigma \mathbf{n})_{\tau}$ on the boundary $\Gamma_{4}$ at different instants $t \in[0,1]$. In each instant, in the same graphic, we can see the tangential stress for the three different boundary conditions considered. We can remark that from $t=0.1 \mathrm{~s}$ the tangential stress obtained for the Coulomb law reaches the bound 0.015 in positive and negative directions. Before $t=0.1 s$ the tangential stress is almost positive. In Figure 7, we plot the tangential velocity on the boundary $\Gamma_{4}$ at the same instants $t \in[0,1]$ considered in Figure 6. Here, we can confirm that, for $t<0.1 s$, there is no negative tangential velocity on $\Gamma_{4}$ for the Coulomb case. Figures 819 are similar to Figures 6 [7, but for instants $t \in[1,6]$. 
Tangential stress on boundary $\Gamma_{4}(T=0.000 s)$

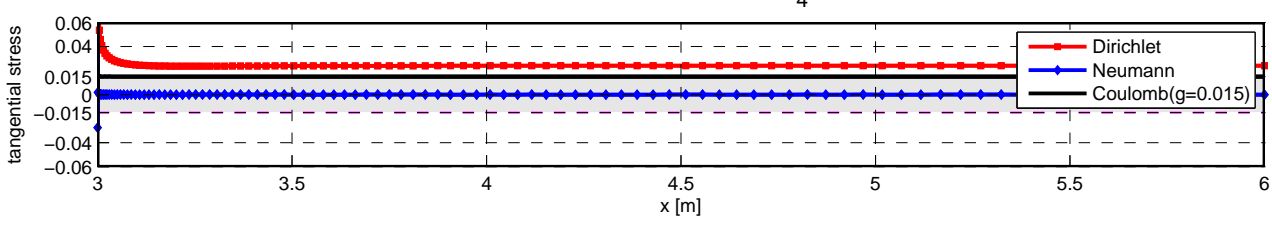

Tangential stress on boundary $\Gamma_{4}(T=0.067 \mathrm{~s})$

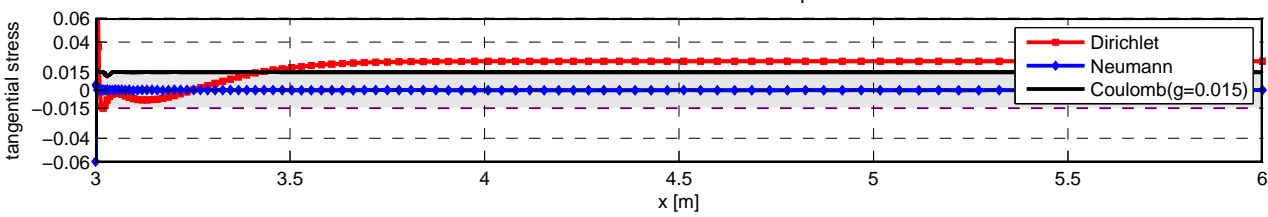

Tangential stress on boundary $\Gamma_{4}(T=0.100 \mathrm{~s})$

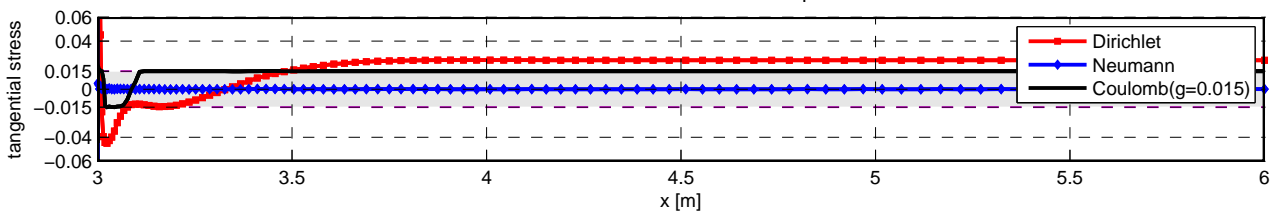

Tangential stress on boundary $\Gamma_{4}(T=0.200 \mathrm{~s})$

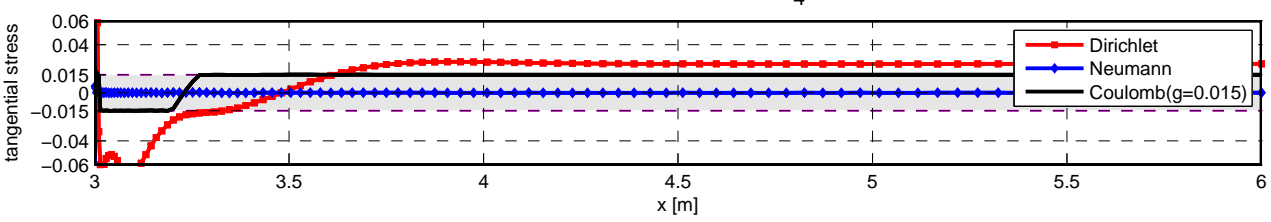

Tangential stress on boundary $\Gamma_{4}(T=0.400 \mathrm{~s})$

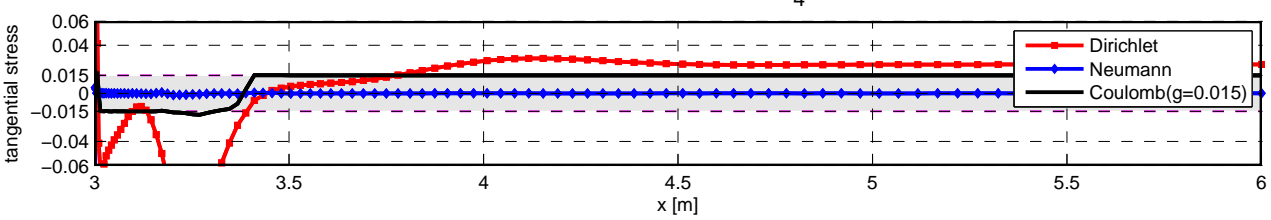

Tangential stress on boundary $\Gamma_{4}(T=0.600 s)$

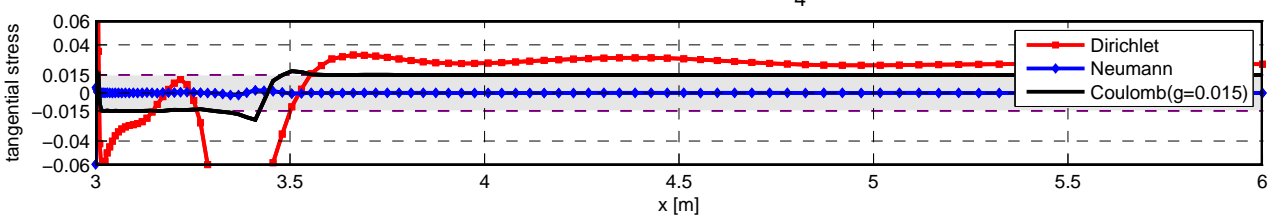

Tangential stress on boundary $\Gamma_{4}(\mathrm{~T}=1.000 \mathrm{~s})$

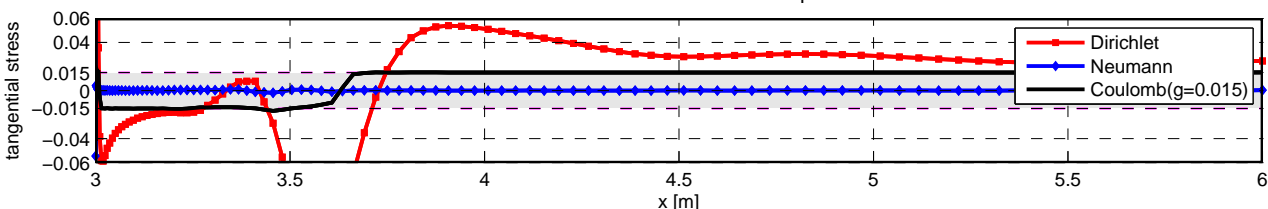

Figure 6. Tangential stress $(\sigma \mathbf{n})_{\tau}$ on $\Gamma_{4}$ at different instants $t \in[0,1]$. 
Horizontal velocity on boundary $\Gamma_{4}(T=0.000 \mathrm{~s})$

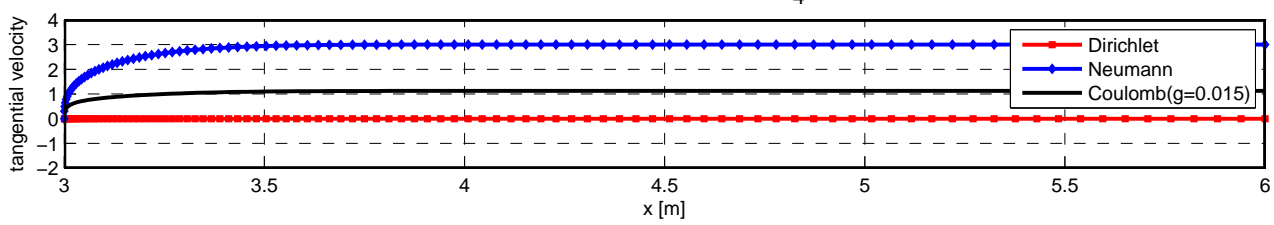

Horizontal velocity on boundary $\Gamma_{4}(T=0.067 \mathrm{~s})$

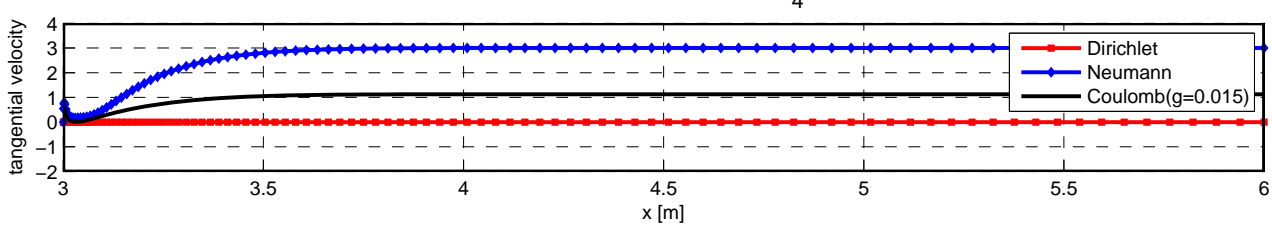

Horizontal velocity on boundary $\Gamma_{4}(T=0.100 \mathrm{~s})$

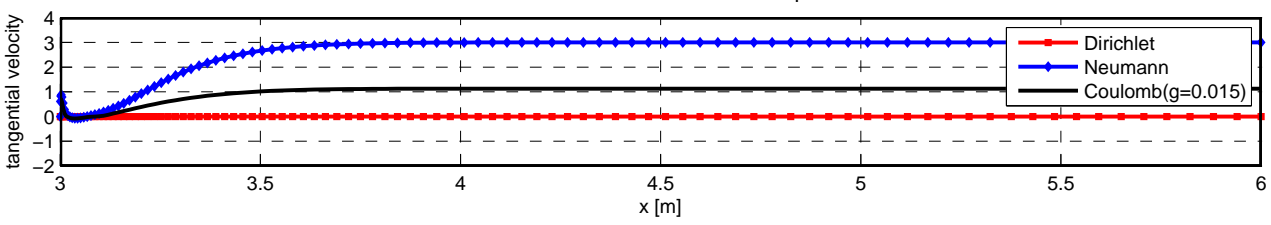

Horizontal velocity on boundary $\Gamma_{4}(T=0.200 \mathrm{~s})$

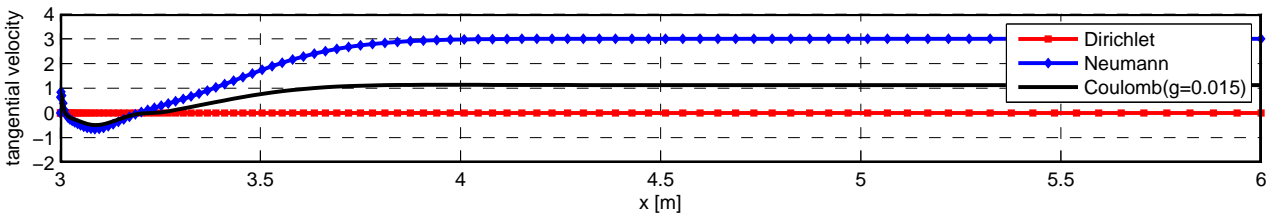

Horizontal velocity on boundary $\Gamma_{4}(T=0.400 \mathrm{~s})$

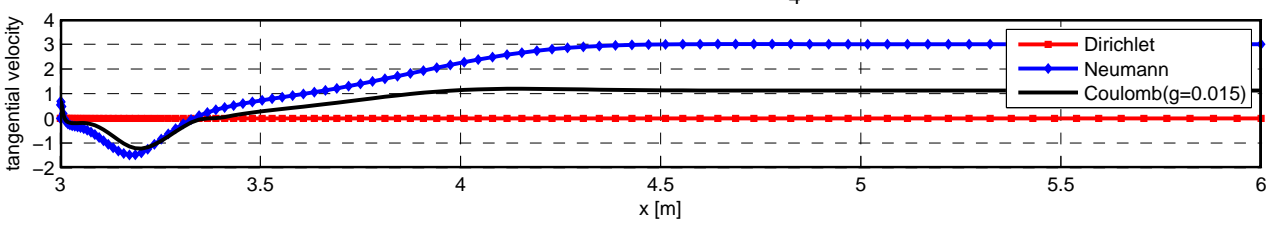

Horizontal velocity on boundary $\Gamma_{4}(T=0.600 \mathrm{~s})$

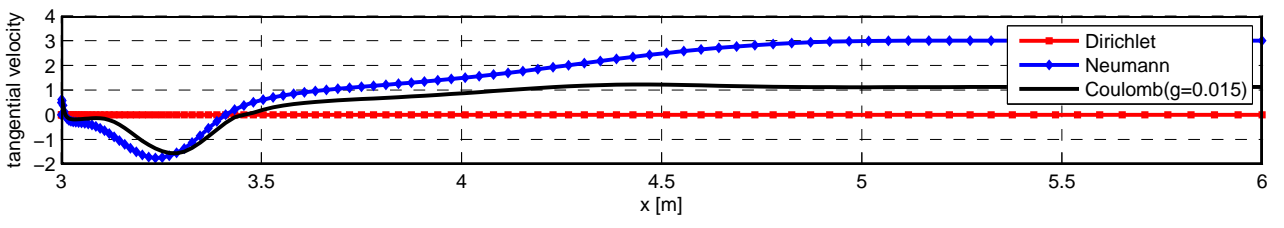

Horizontal velocity on boundary $\Gamma_{4}(T=1.000 \mathrm{~s})$

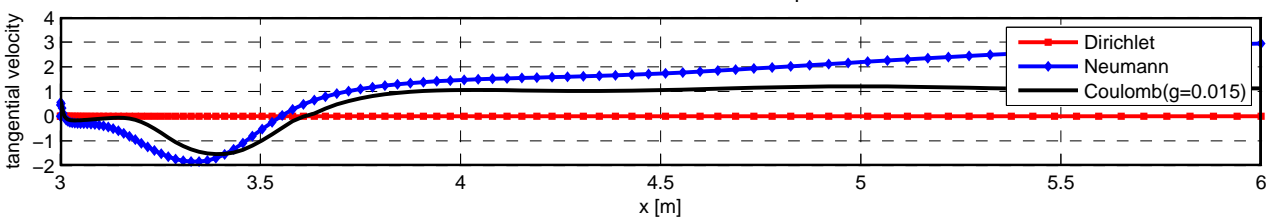

Figure 7 . Tangential velocity field $\mathbf{u}_{\tau}$ on $\Gamma_{4}$ at different instants $t \in[0,1]$. 
Tangential stress on boundary $\Gamma_{4}(T=1.000 \mathrm{~s})$

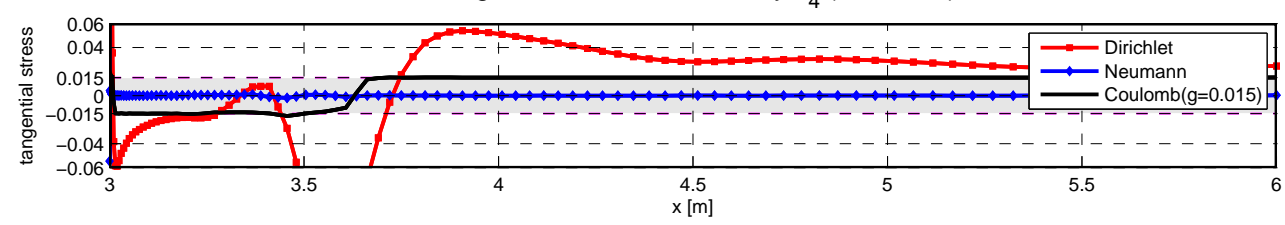

Tangential stress on boundary $\Gamma_{4}(\mathrm{~T}=2.000 \mathrm{~s})$

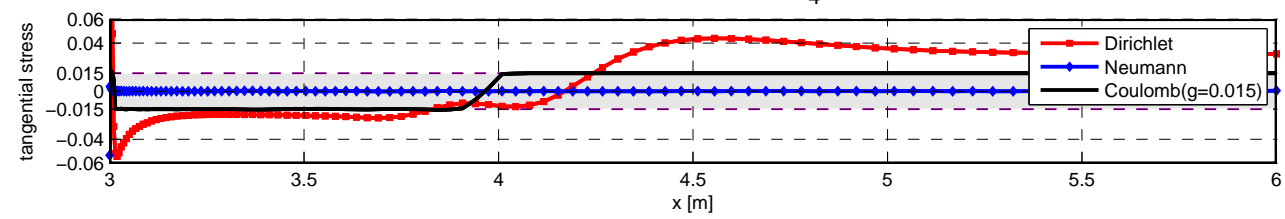

Tangential stress on boundary $\Gamma_{4}(T=3.000 \mathrm{~s})$

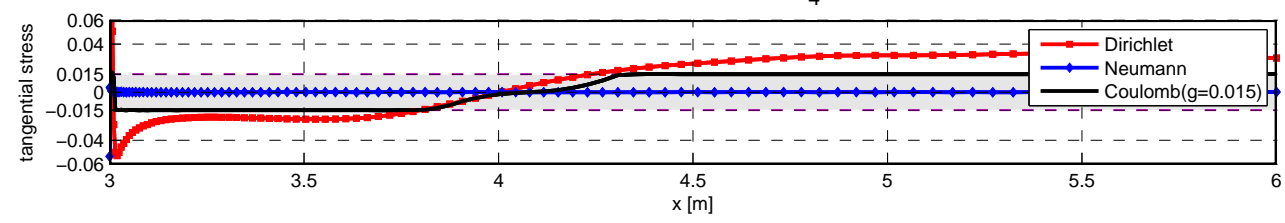

Tangential stress on boundary $\Gamma_{4}(T=4.000 \mathrm{~s})$

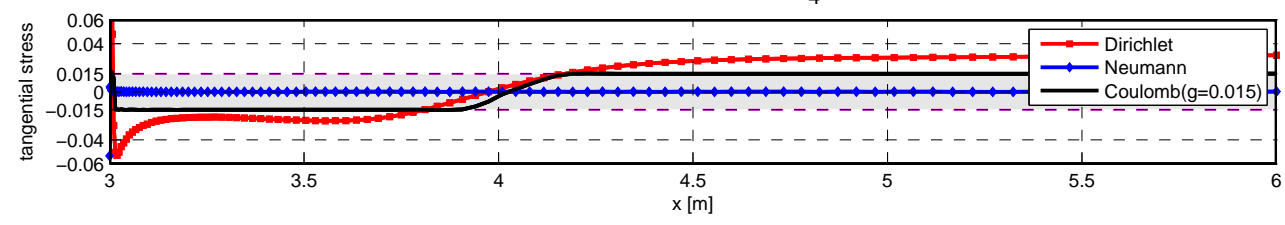

Tangential stress on boundary $\Gamma_{4}(T=5.000 \mathrm{~s})$

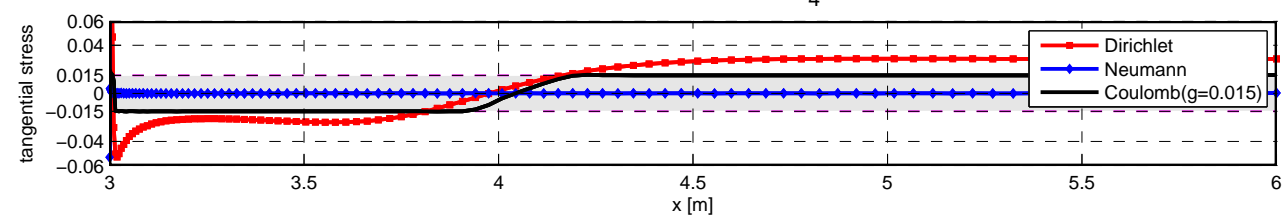

Tangential stress on boundary $\Gamma_{4}(T=6.000 \mathrm{~s})$

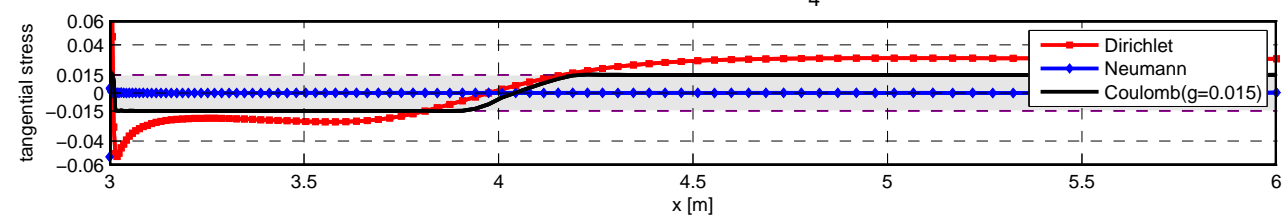

Figure 8. Tangential stress $(\sigma \mathbf{n})_{\tau}$ on $\Gamma_{4}$ at different instants $t \in[1,6]$. 


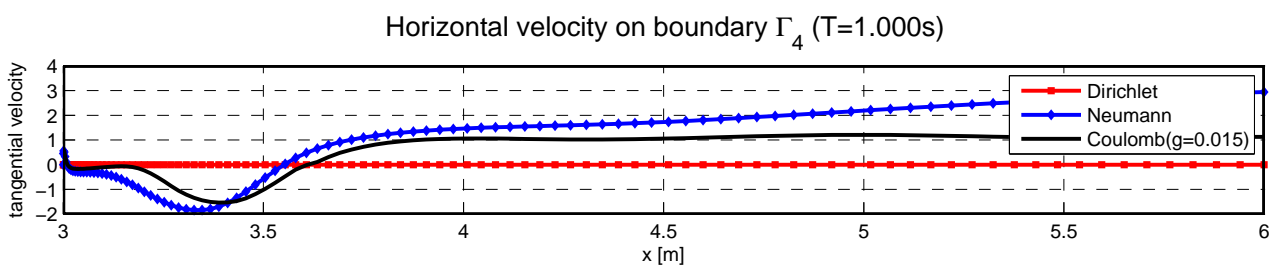

Horizontal velocity on boundary $\Gamma_{4}(T=2.000 \mathrm{~s})$

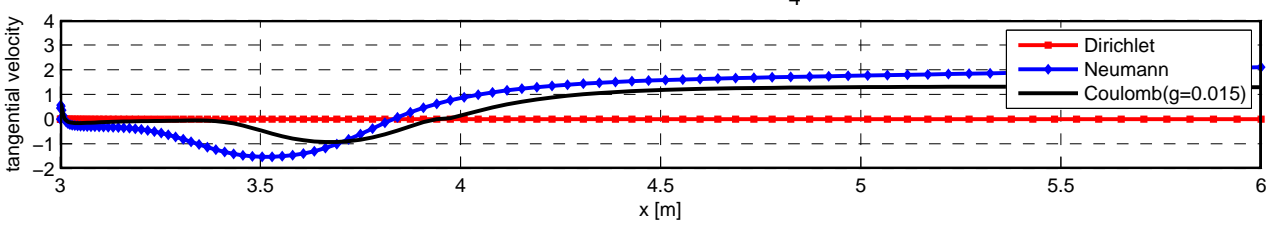

Horizontal velocity on boundary $\Gamma_{4}(T=3.000 \mathrm{~s})$

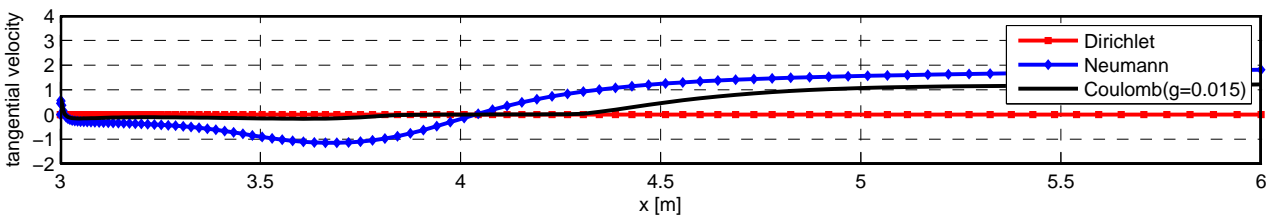

Horizontal velocity on boundary $\Gamma_{4}(T=4.000 \mathrm{~s})$

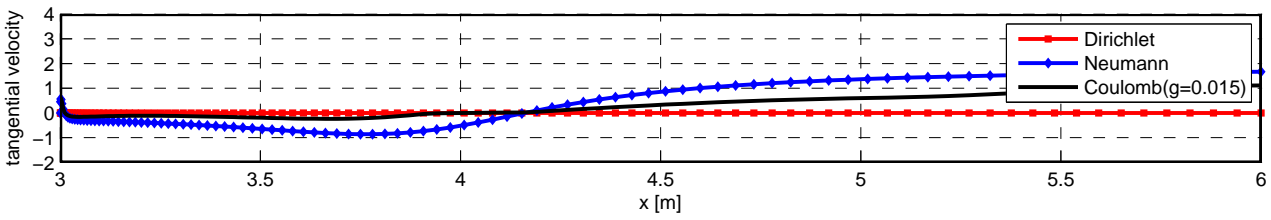

Horizontal velocity on boundary $\Gamma_{4}(T=5.000 \mathrm{~s})$

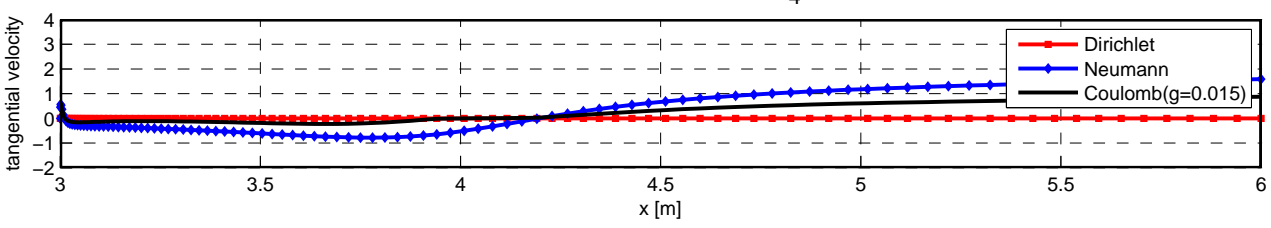

Horizontal velocity on boundary $\Gamma_{4}(T=6.000 \mathrm{~s})$

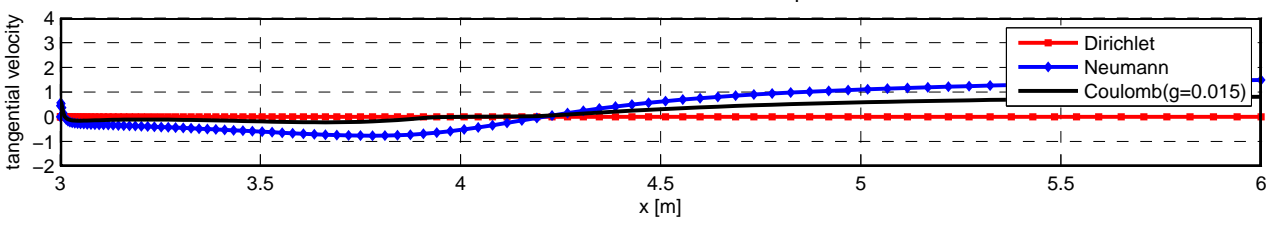

Figure 9. Tangential velocity field $\mathbf{u}_{\tau}$ on $\Gamma_{4}$ at different instants $t \in[1,6]$. 
4.2. Results for the flow around a cylindrical obstacle in a rectangular channel. This numerical test has been performed in the documentation of the FreeFem++ program (see [8]) for the case of the Dirichlet boundary condition on $\Gamma_{4}$. For the case of the Coulomb law we consider two cases: the first one with $g=0.07$ and the second with $g=0.2$. The first value was chosen below the maximum tangential stress obtained for the Dirichlet boundary condition at $t=0$. The second value is greater that the maximum stress at $t=0$ (then it is not active for the initial condition, but it becomes active afterward during the simulation). In Figures 10 11, we present the velocity field obtained for the four different boundary conditions at $t=0$ and $t=2 s$. In Figure 10, we remark that the solutions of the Stokes problem, subplots a) and d) are the same, since the Coulomb law is reduced to the Dirichlet case with our choice of $g$. In Figure 11, the four velocity fields are different.

In Figures 12 14, we plot the tangential velocity field and the tangential stress on the boundary $\Gamma_{4}$ for the four different boundary conditions considered. In the first one, we present the dependence of both quantities with respect to the angular position around the cylindrical obstacle at $t=2 s$. In the second one, we give the evolution in time of the maximum of the same quantities for $t \in[0,2]$. Finally, in Figure 14, we show a zoom of the same evolution for $t \in[0,0.2]$. 

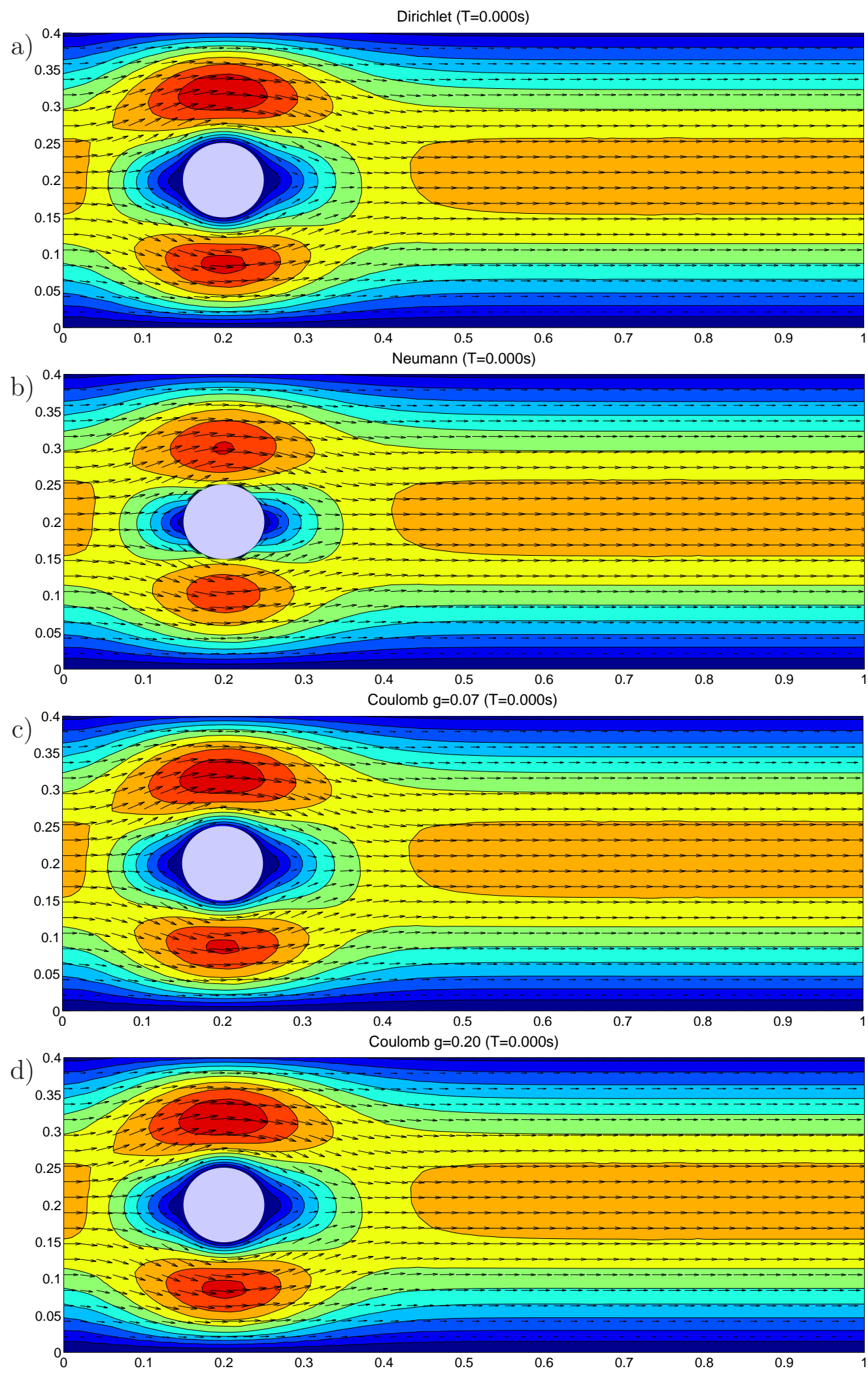

FIGURE 10. Velocity field at $t=0$, obtained as the solution of Stokes equation with different boundary conditions on $\Gamma_{4}$ (with $R e=100$ ): a) Homogeneous Dirichlet boundary condition b) Neumann boundary condition c) Coulomb boundary condition with $g=0.07 \mathrm{~d}$ ) Coulomb boundary condition with $g=$ 0.20 

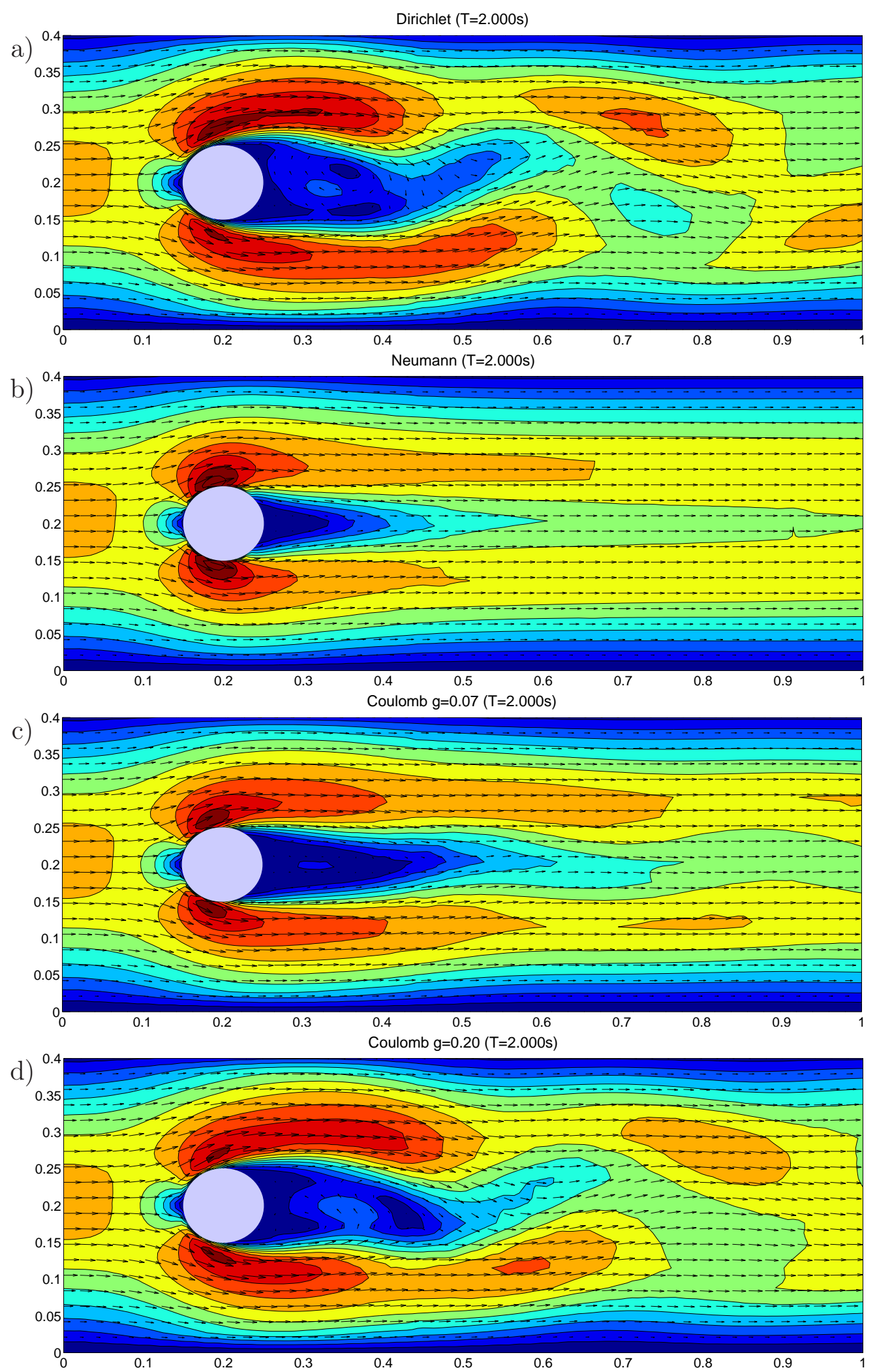

FiguRE 11. Velocity field at $t=2 s$, obtained as the solution of Navier-Stokes equation with the four boundary conditions on $\Gamma_{4}$ (with $R e=100$ ): a) Zero Dirichlet boundary condition b) Neumann boundary condition c) Coulomb boundary condition with $g=0.07 \mathrm{~d}$ ) Coulomb boundary condition with $g=$ 0.20 

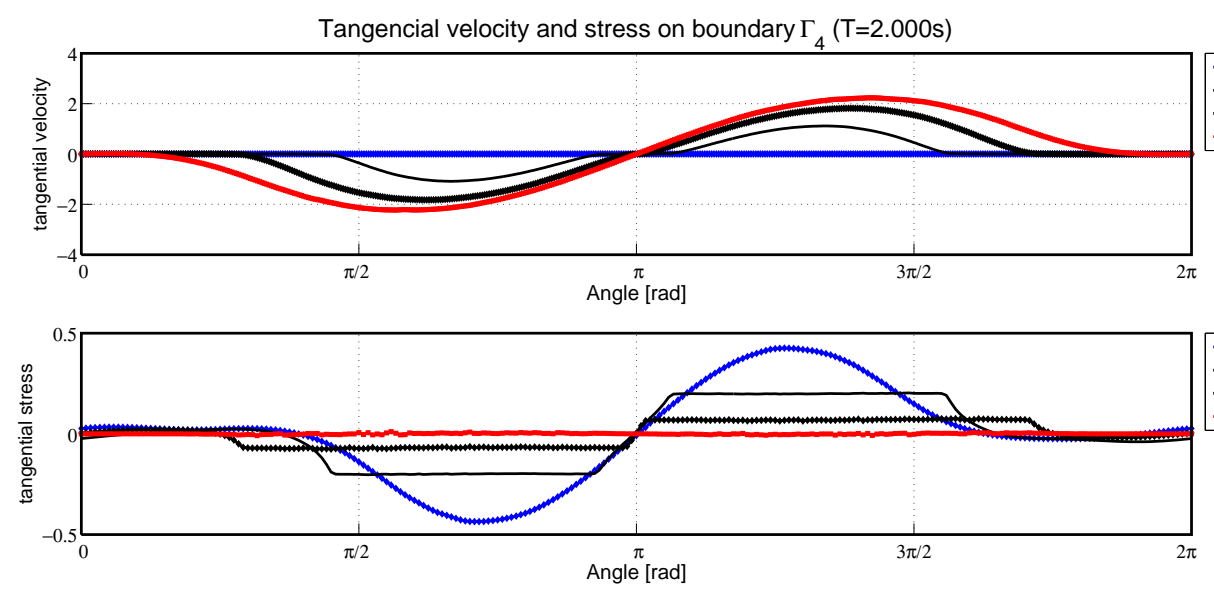

$\rightarrow$ Dirichlet

- Coulomb $(g=0.20)$ 千Coulomb(g=0.07) - Neumann

FiguRE 12. Tangential velocity $\mathbf{u}_{\tau}$ and tangential stress $(\sigma \mathbf{n})_{\tau}$ on the boundary $\Gamma_{4}$ of the cylinder.
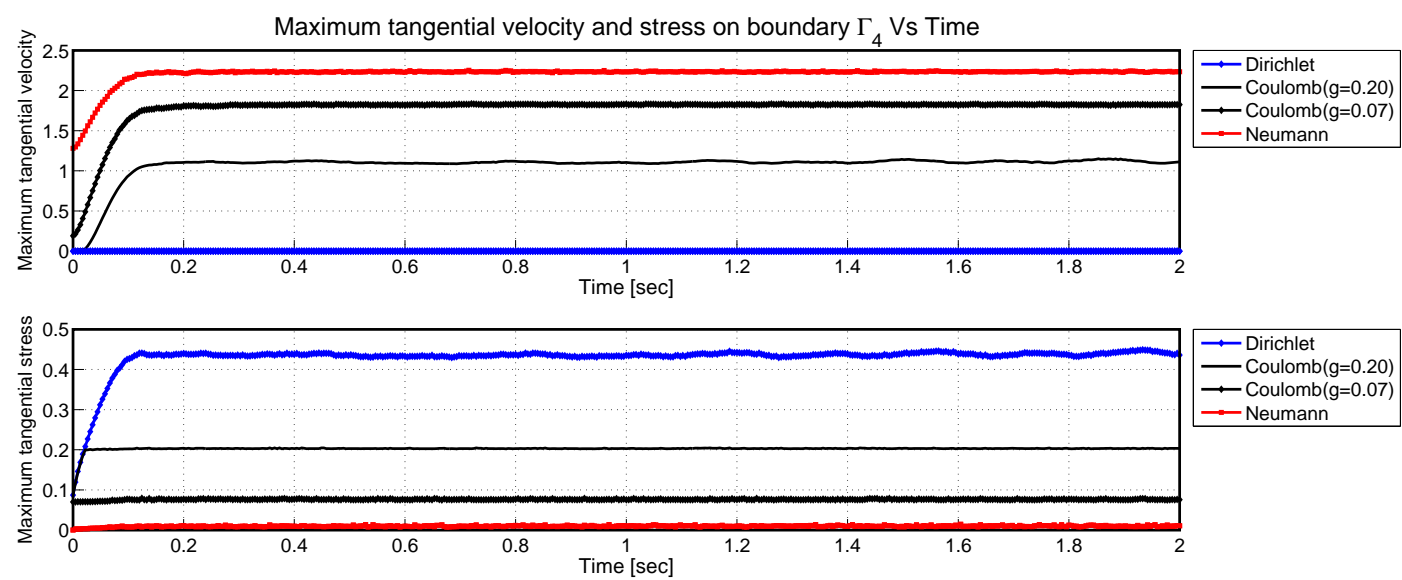

二- Dirichlet -Coulomb $(g=0.20$ $\rightarrow$ Coulomb $(\mathrm{g}=0.07)$ $\rightarrow$ Neumann

FIGURE 13. Graphics in time $t$ of the tangential velocity $\mathbf{u}_{\tau}$ and the tangential stress $(\sigma \mathbf{n})_{\tau}$ on the boundary $\Gamma_{4}$ of the cylinder.
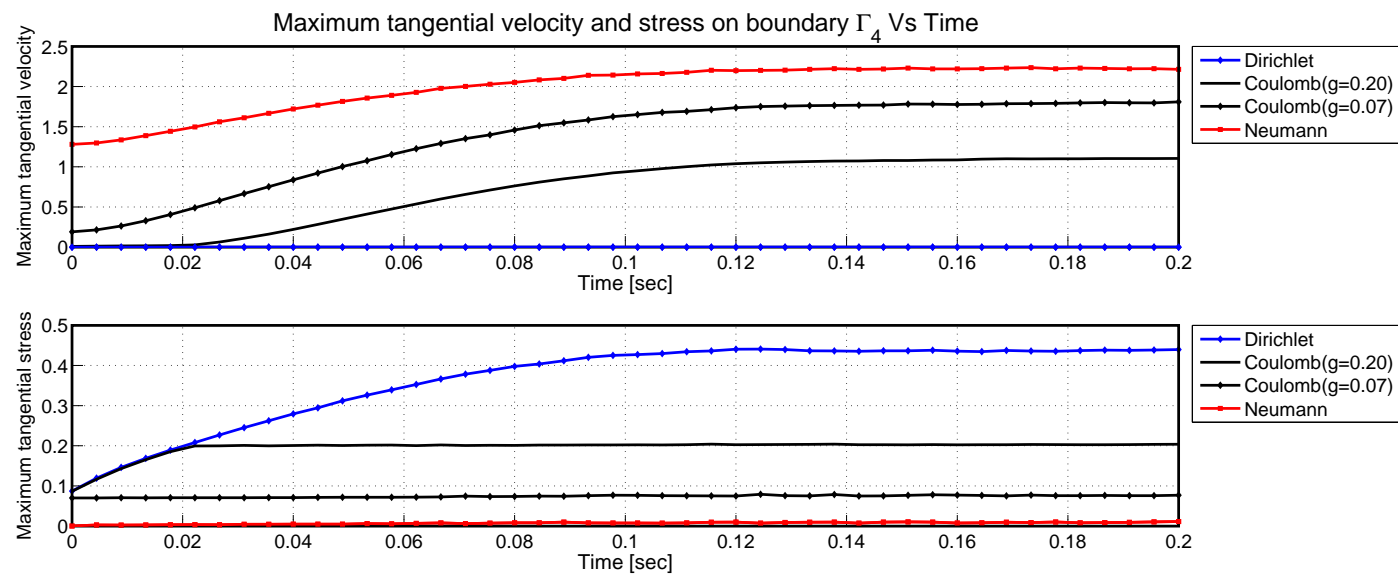

千-Dirichlet - Coulomb $(\mathrm{g}=0.20)$ - Neumann

FigurE 14. Graphics of the tangential velocity $\mathbf{u}_{\tau}$ and the tangential stress $(\sigma \mathbf{n})_{\tau}$ on the boundary $\Gamma_{4}$ of the cylinder for $t \in[0,0.2]$. 


\section{ACKNOWLEDGEMENTS}

J. San Martín was partially supported by PFBasal-03 (CMM), PFBasal-01 (CeBiB) projects. T. Takahashi was partially supported by the ANR research project IFSMACS (ANR-15CE40-0010).

\section{REFERENCES}

[1] H. Brezis, Analyse fonctionnelle, Collection Mathématiques Appliquées pour la Maîtrise. [Collection of Applied Mathematics for the Master's Degree], Masson, Paris, 1983. Théorie et applications. [Theory and applications].

[2] C. Conca, J. San Martín, And M. Tucsnak, Existence of solutions for the equations modelling the motion of a rigid body in a viscous fluid, Comm. Partial Differential Equations, 25 (2000), pp. 1019-1042.

[3] G. Duvaut and J. L. Lions, Les inéquations en méchanique et en physique, Dunod, Paris, France, 1972. (in French).

[4] D. GÉrard-Varet and M. Hillairet, Existence of weak solutions up to collision for viscous fluidsolid systems with slip, Comm. Pure Appl. Math., 67 (2014), pp. 2022-2075.

[5] D. Gérard-Varet, M. Hillairet, and C. Wang, The influence of boundary conditions on the contact problem in a 3D Navier-Stokes flow, J. Math. Pures Appl. (9), 103 (2015), pp. 1-38.

[6] V. Girault and P.-A. Raviart, Finite element approximation of the Navier-Stokes equations, Springer-Verlag, Berlin, 1979.

[7] R. GLowinski, Numerical methods for nonlinear variational problems, Springer series in computational physics, Springer, New York, 1984.

[8] F. Hecht, New development in FreeFem++, J. Numer. Math., 20 (2012), pp. 251-265.

[9] O. Ladyzhenskaya, The mathematical theory of viscous incomprehensible flow, Gordon and Breach Science Publishers, New York, 1969.

[10] J.-L. Lions, Quelques méthodes de résolution des problèmes aux limites non linéaires, Dunod; GauthierVillars, Paris, 1969.

[11] J.-L. Lions and E. Magenes, Non-homogeneous boundary value problems and applications. Vol. I, Springer-Verlag, New York, 1972. Translated from the French by P. Kenneth, Die Grundlehren der mathematischen Wissenschaften, Band 181.

[12] O. Pironneau, On the transport-diffusion algorithm and its applications to the Navier-Stokes equations, Numer. Math., 38 (1982), pp. 309-332.

[13] J. San Martín, J.-F. Scheid, and L. Smaranda, A modified Lagrange-Galerkin method for a fluidrigid system with discontinuous density, Numer. Math., 122 (2012), pp. 341-382.

[14] J. San Martín, J.-F. Scheid, T. Takahashi, and M. Tucsnak, Convergence of the LagrangeGalerkin method for the equations modelling the motion of a fluid-rigid system, SIAM J. Numer. Anal., 43 (2005), pp. 1536-1571 (electronic).

[15] J. A. San Martín, V. Starovoitov, and M. Tucsnak, Global weak solutions for the two-dimensional motion of several rigid bodies in an incompressible viscous fluid, Arch. Ration. Mech. Anal., 161 (2002), pp. $113-147$.

[16] E. SÜLI, Convergence and nonlinear stability of the Lagrange-Galerkin method for the Navier-Stokes equations, Numer. Math., 53 (1988), pp. 459-483.

[17] R. Temam, Navier-Stokes equations, North-Holland Publishing Co., Amsterdam, third ed., 1984. Theory and numerical analysis, With an appendix by F. Thomasset. 